\title{
Fluorescence Techniques to Study Lipid Dynamics
}

\author{
Erdinc Sezgin and Petra Schwille \\ Biophysics Group, BIOTEC, TU Dresden, 01307 Dresden, Germany \\ Correspondence: schwille@biotec.tu-dresden.de
}

Biological research has always tremendously benefited from the development of key methodology. In fact, it was the advent of microscopy that shaped our understanding of cells as the fundamental units of life. Microscopic techniques are still central to the elucidation of biological units and processes, but equally important are methods that allow access to the dimension of time, to investigate the dynamics of molecular functions and interactions. Here, fluorescence spectroscopy with its sensitivity to access the single-molecule level, and its large temporal resolution, has been opening up fully new perspectives for cell biology. Here we summarize the key fluorescent techniques used to study cellular dynamics, with the focus on lipid and membrane systems.

$T^{\circ}$ elucidate cellular processes in their native dynamic environment has been one of the main issues in cell biology over the past decades. The lack of appropriate techniques has long been the main limiting step for the research on dynamic systems, because it was impossible to acquire real time information with the well-known biochemical techniques. The key challenge in dynamically observing biological systems is to combine the ability to resolve moderate to very low concentrations of molecules-because they are simply limited in living cells_-on relevant timescales. Relevant timescales in cell biology can be minutes and hours, on a systemic level of cell metabolism, down to the microsecond and even nanosecond regime in which molecular and intramolecular rearrangements take place. With respect to lipidic systems, relevant dynamics range from the local movements of lipids by diffusion to the mechanical transformations of whole membranes, spanning several orders of magnitude in time to be covered. Like for other cellular processes, the investigation of lipids and membranes also in general benefited greatly from the introduction of fluorescence microscopy and spectroscopy to biology. After the 1960s, great technological inventions based on the phenomenon of fluorescence were made, such as confocal microscopy, fluorescence recovery after photobleaching (FRAP), fluorescence correlation spectroscopy (FCS), Förster resonance energy transfer (FRET), total internal reflection fluorescence (TIRF), and two-photon microscopy, that not only revolutionized imaging but also yielded access to dynamics on previously inaccessible timescales. Another very big step was certainly taken after the introduction

Editor: Kai Simons

Additional Perspectives on The Biology of Lipids available at www.cshperspectives.org

Copyright (C) 2011 Cold Spring Harbor Laboratory Press; all rights reserved; doi: 10.1101/cshperspect.a009803

Cite this article as Cold Spring Harb Perspect Biol 2011;3:a009803 
of fluorescent proteins, which again accelerated the use of these techniques in living cells and organisms. Nowadays, the technical advancements of fluorescence-based methods allow us to explore systems as small as single molecules with temporal resolution down to the nanoseconds regime. Lately, even the resolution limit of optical microscopy, for a long time being one of the fundamental barriers in elucidating cellular processes, has been overcome by smart applications of the phenomenon of fluorescence.

This article aims at giving a short overview on mainly fluorescence-based methods that have in recent years propelled lipid and membrane research to fully new levels. We will give a short introduction to the modern fluorescence technology in general, referring to the techniques that allow addressing dynamics. A particular focus will be on fluorescence correlation spectroscopy, a technique that our lab works primarily on, but other important methods will also be discussed, including their promises, achievements, and caveats.

\section{FLUORESCENCE TO STUDY LIPID DYNAMICS}

The attempt to visualize the "living units" has progressed remarkably after Hooke's Micrographia. Starting from a simple light source, a mechanical stage, and up to three glass lenses, microscopy nowadays culminated in so-called super-resolution techniques with particle localization accuracies down to the nanometer range. Certainly, the involvement of the phenomenon of fluorescence is one of the biggest steps in this long journey.

Fluorescence is such a ubiquitous phenomenon that it is impossible to speculate about its first systematic observation. The first reported documentation of fluorescence is thought to be Nicolas Monardes' observation of wood extract. In 1845, John Herschel observed the fluorescent property of quinine sulphate which is believed to be the onset of modern fluorescence spectroscopy. After many more observations by several light philosophers in the 19th century, it was Stokes who actually termed this phenomenon "fluorescence" in 1838. The first application in biology was probably in 1914 Stanislav von Provazek who used fluorescence as a cell stain. August Koehler and Oskar Heimstadt were reportedly the first scientists who performed fluorescence microscopy in early 1900s. Today, a century later, fluorescence imaging and microscopy is one of the most powerful tools in the visualization and dynamic analysis of living structures, especially following the discovery of fluorescent proteins as cloneable markers, and the invention and widespread use of confocal microscopy. Minsky, its inventor, patented the idea of confocal microscopy already in the 1950s, and about 20 years later, the first commercial confocal microscopes appeared. Since then, many researchers and optical engineers step by step improved the technical realization (Brakenhoff et al. 1979; Davidovi and Egger 1973; Egger and Petran 1967; Hamilton and Wilson 1986; Sheppard and Wilson 1979). The rapid developments in laser and detector technology, along with the onset of fiber optics certainly helped in the rapid dissemination of confocal microscopy into cell biology laboratories around the world (Amos and White 2003).

When light interacts with matter, many photophysical phenomena may occur. Some molecules absorb light at a particular wavelength, whereas others predominantly scatter the light. On absorption, the molecules undergo vibrational relaxation on timescales between $10^{-14}$ and $10^{-12} \mathrm{sec}$, and then return to ground state, either by emitting a photon at a longer wavelength after $10^{-9}$ to $10^{-7}$ nsec, which is called fluorescence, or nonradiatively. Less probably, the molecules can jump to the quantummechanically forbidden triplet state or molecules transfer their energy to other molecules, by quenching or resonant energy transfer. After the molecules undergo the triplet state, they return to the ground state either by emitting light in longer time ranges than fluorescence or nonradiatively.

In the following sections, we will briefly touch on the task of fluorescently labeling lipids to be investigated, and then discuss, one by one, the most powerful biophysical techniques to study lipids and membranes in real time, along with some of their relevant applications. 
Fluorescent Probes to Study Lipid Dynamics

After the invention of green fluorescent protein (GFP) as the first truly genetic fluorescent probe, visualizing proteins in their native environment became much more straightforward. From the perspective of the membrane researcher, this significantly improved our understanding of membrane proteins and their dynamics, but could help only marginally in better elucidating the functional dynamics of lipids. The first report on labeling lipids in living cells used azide-alkyne to label alkyne containing PA (Schultz et al. 2010). Besides this direct labeling, coupling the synthetic fluorescence molecules to lipids in vitro, and then reconstitute them to the cell membrane is getting more common in lipid field, enforcing the use of fluorescence also in lipid biology. Synthetic dye coupling has many advantages compared to fluorescent proteins, which nowadays represent the main strategy in protein labeling. First of all, one has theoretically a large choice of organic dyes in terms of their optical characteristics. It is possible, for instance, to use a far red dye; however, there is not yet a well-established monomeric far red protein. Second, the quantum efficiency and brightness of most of the organic dyes are higher than for fluorescent proteins. Cholesterol (Boldyrev et al. 2007; Holtta-Vuori et al. 2008; Marks et al. 2008; Oreopoulos and Yip 2009), Sphingomyelin (Marks et al. 2008; Eggeling et al. 2009; Tyteca et al. 2010), GM1 (Coban et al. 2007; Eggeling et al. 2009; Mikhalyov et al. 2009), PC, and PE (Baumgart et al. 2007; Juhasz et al. 2010) are some of the lipids that are often conjugated to organic dyes. Additionally, fluorescently labeled membrane-binders, like choleratoxin, are used to label, for example, the GMs on the cell surface (Middlebrook and Dorland 1984). However, taking into account that organic fluorophores are in comparison much larger handicaps to small lipid molecules than they are to proteins, and that the relatively tight packing of lipids in a membrane might be more easily disturbed by labeled lipids than in the case of soluble proteins, a careful control of the possible influence of labels on the functionality of lipids is of utmost importance.
Besides fluorescent lipid conjugates, there are some lipophilic fluorescent molecules frequently used to yield information on a specific lipid environment. They efficiently and selectively penetrate into lipid membranes, and to some extent even reflect on their physical properties, like viscosity, order, $\mathrm{pH}$, or water content. DiO, DiD, DiI, Laurdan, and NAP are the lipophilic dyes most commonly used to visualize the lipid environment (Baumgart et al. 2007). Although the Di family of dyes is phase-preferring probes preferring either liquid-ordered $\left(\mathrm{L}_{\mathrm{o}}\right)$ phase or liquid-disordered $\left(\mathrm{L}_{\mathrm{d}}\right)$ phase in a specific setting, Laurdan has a different property. It partitions equally in both phases, but its emission spectrum changes according to the polarity of the membrane environment. Providing that $\mathrm{L}_{\mathrm{d}}$ phase is more aqueous than $\mathrm{L}_{\mathrm{o}}$ phase, on excitation the dye consumes some of its energy to reorient the water molecules in $\mathrm{L}_{\mathrm{d}}$ phase, which shifts the emission to the red spectral region (emission maximum of $490 \mathrm{~nm}$ ), whereas it is more blue shifted in $\mathrm{L}_{\mathrm{o}}$ region (emission maximum of $440 \mathrm{~nm}$ ). According to the ratio of fluorescence intensity in the blue-shifted $\left(\mathrm{L}_{\mathrm{o}}\right.$ phase) and the red-shifted region ( $\mathrm{L}_{\mathrm{d}}$ phase), one can calculate an order indicative value called generalized polarization (GP) calculated as

$$
\mathrm{GP}=\frac{I_{440}-I_{490}}{I_{440}+I_{490}},
$$

where $I_{x}$ denotes the intensity at wavelength of $x$.

In addition to generalized polarization, fluorescence anisotropy is another important phenomenon that can be exploited to monitor rotational diffusion of the molecules by using the polarization of light. Because rotational diffusion is very sensitive to the size of molecules, binding constants can be efficiently derived from fluorescence anisotropy measurements. There have been comparative studies on the feasibility of several dyes for fluorescence anisotropy. Alexa and Oregon dyes conjugates with biological molecules (e.g., lipids), for instance, were found to be suitable for this method (Rusinova et al. 2002). Additionally, NBD and DHP lipid conjugates were used for fluorescence anisotropy to detect rafts in living cells (Gidwani et al. 2001). Laurdan generalized polarization 
and fluorescence anisotropy were compared elsewhere (Engelke et al. 2001).

Besides lipid probes for the plasma membrane, there are also some tools to probe other lipidic environments in the cell, such as lipid droplets (Thiele and Spandl 2008). New fluorescent lipids were developed to visualize the intracellular and membrane lipids in their native environment without any external fluorescent labels (Kuerschner et al. 2005; Spandl et al. 2009).

\section{Confocal Microscopy}

Confocal microscopy may easily be the most widely applied imaging technique in cell and molecular biology field because it allows live cell imaging with high spatial and temporal resolution, as well as optical sectioning and 3D reconstruction of images. To start with the techniques for cell dynamics, confocal microscopy should therefore be briefly mentioned because it forms the basis (and often the gold standard) for most of the other techniques.

The confocal concept evolved as an alternative to wide-field microscopy. For wide-field microscopy, the so-called Koehler illumination guarantees a homogeneous illumination of the whole sample, which is then detected by area detectors. In contrast to this, confocal illumination occurs only at a resolution-limited point, which can then be sequentially scanned in three dimensions throughout the sample. As a technical difference, coherent light sources (lasers) are usually employed in confocal microscopy, whereas incoherent lamps are still mostly used in wide-field microscopy. However, the basic difference between wide-field and confocal microscopies is a so-called pinhole aperture which eliminates the out-of-focus light in the image plane, being the main source of background in wide field. The minimal size of the confocal illumination volume, and therefore the resolution that can be reached in confocal microscopy is usually determined by the so-called Rayleigh criterion. Here, resolution of the wide field is defined as the shortest distance $d$ between two optically separable points:

$$
d=\frac{0.61 \times \lambda}{\mathrm{NA}}
$$

where $\lambda$ is the wavelength and NA is the numerical aperture of the objective. When the advantage of selective detection (pinhole) and selective illumination (diffraction limited spot by coherent light source) are applied, the resolution reaches a better point:

$$
d=\frac{0.4 \times \lambda}{\mathrm{NA}}
$$

Taking above equation into consideration, the theoretical resolution of a confocal system with an NA of 1.4, at a wavelength of $500 \mathrm{~nm}$ should be $\sim 160 \mathrm{~nm}$. However, all theoretical calculations consider a perfect optical system and a pinhole of a laser spot size (i.e., Airy disc size). Yet, there are many aberrations caused by imperfect optics such as spherical aberrations, chromatic aberrations, astigmatism, comma etc. Moreover, pinhole size can never be as small as laser spot size. The biggest problem in confocal microscopy is, however, the large discrepancy between lateral $(x-y)$ and axial resolution, resulting in image stacks that are usually quite blurred in the $z$ dimension.

Axial resolution is given by

$$
d_{\mathrm{z}}=\frac{1.4 \times \lambda \times n}{\mathrm{NA}^{2}},
$$

where $n$ is the refractive index of the medium. The axial resolution is usually three to five times worse than lateral resolution.

The limitation in axial resolution is a minor problem for pure membrane systems with little to no contribution of fluorescence light coming from the solution above and below the membrane. Thus, confocal microscopy has been particularly useful on supported membranes or giant unilamellar vesicles (GUVs) (Korlach et al. 1999). On the other hand, for the study of cellular membranes with their rather high background from cellular autofluorescence and labeled molecules that cannot easily be retained at the cell surface (e.g., because of endocytosis), limited $z$ resolution can be a significant technical problem in studying lipid dynamics. For this reason, other illumination strategies established for fluorescence microscopy, such as total internal reflection (TIR), are 
becoming increasingly popular for lipid and membrane research.

\section{Total Internal Reflection Fluorescence (TIRF) Microscopy}

Total internal reflection is a well-known phenomenon in everyday life. When a light beam passes from a medium with a refractive index $n_{1}$ to the other medium with a refractive index $n_{2}$, the relationship between the angle of incidence $\alpha_{1}$ and the angle of refraction $\alpha_{2}$ is determined by Snell's law:

$$
n_{1} \sin \alpha_{1}=n_{2} \sin \alpha_{2} \text {. }
$$

When $n_{1}>n_{2}$ (e.g., when light is propagating from water or glass into air), there exists a critical $\alpha_{1}$ angle that would render the refraction angle to be $90^{\circ}$ (i.e., parallel to the interface). At incidence angles equal to or greater than this critical angle, light cannot cross the boundary, and is internally reflected. This phenomenon is called total internal reflection (TIR). The critical angle $\left(\alpha_{c}\right)$ is determined by the refractive indices of the media:

$$
\alpha_{c}=\sin ^{-1}\left(n_{2} / n_{1}\right) .
$$

Although the light ray is totally reflected, its electrical field creates an evanescent wave whose intensity decreases exponentially in the axial direction (Fig. 1):

$$
I(z)=I(0) e^{-z / d}
$$

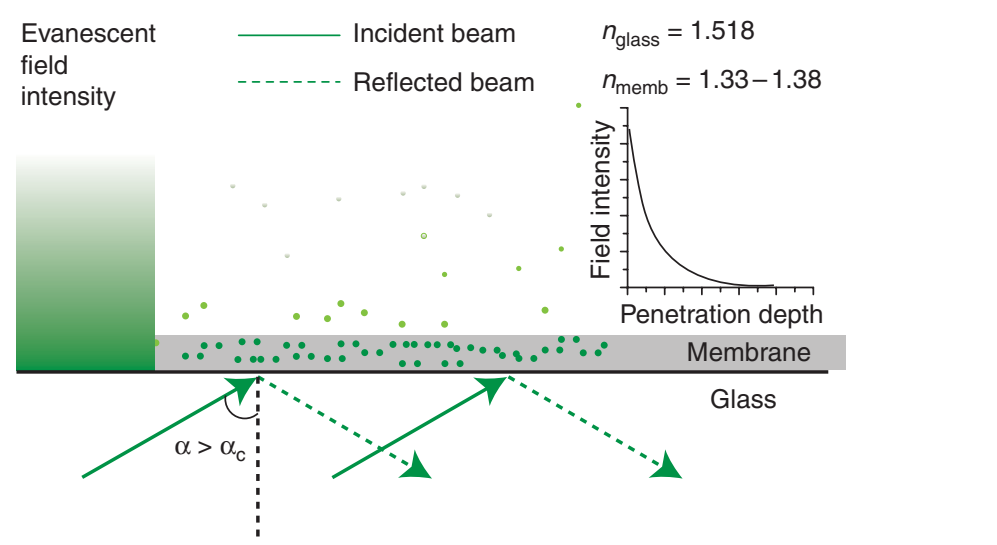

Figure 1. Evanescent wave and TIRF. where $d$ is defined as

$$
d=\left(\frac{\lambda}{4 \pi}\right)\left(n_{1}^{2} \sin ^{2} \alpha_{1}-n_{2}^{2}\right)^{-1 / 2},
$$

and $\lambda$ is the wavelength. The principle is schematically depicted in Figure 1.

The most remarkable feature of TIR illumination for membrane research is that the evanescent wave illuminates only a limited distance $(\sim 100 \mathrm{~nm})$ along the $z$-axis, which reduces background coming from molecules above the surface substantially, thereby increasing the axial resolution and the signal-to-noise levels. The detailed physics behind TIR can be accessed from references Axelrod (2008) and Axelrod et al. (1983).

There are currently two methods to realize this illumination mode for fluorescence microscopy. The first, simpler, method is to use a high-NA TIRF objective. The light beam entering the objective is focused at the back focal plane of the objective with a displacement from the optical axis, such that it reaches the surface above the critical angle. Surface fluorescence is collected by the same objective and can be detected in the usual way.

The necessity for using a high-NA objective can be understood by considering that

$$
\mathrm{NA}=n \sin \theta .
$$

Then, Equation 8 can be rewritten as

$$
d=(\lambda / 4 \pi)\left(\mathrm{NA}^{2}-n_{2}^{2}\right)^{-1 / 2} .
$$


When NA $<n_{2}, d$ is imaginary, which means light is refracted and TIR is lost. That is why the NA of TIRF objectives should be higher than the refractive index of the sample medium. A living cell has a refractive index of 1.33-1.38. Many current TIRF objectives thus have a NA of 1.45 , which creates a penetration depth of the evanescent field of $82 \mathrm{~nm}$ at $488 \mathrm{~nm}$ excitation just above the critical angle.

The second way to create an evanescent wave is to use a prism. In this case, the sample is located between the prism and objective. The illumination is performed through the prism, while the objective collects the emission and transfers the signal to the camera. This decoupling of illumination and detection can be quite useful to create large illumination areas, but is less comfortable than objectivebased TIR, which can be easily realized in any fluorescence microscope.

Further advantages of prism-based TIR are lower background and a better control on angle and polarization. It is also easy to set up for two colors. On the other hand, the free access to one side of the sample, safety of lasers, ease to use with cell culture plates can be counted as the advantages of objective-based system.

TIRF can be coupled to other techniques as a specific illumination mode when $\operatorname{good} z$ resolution is crucial. It has been combined with FCS, FRET, FRAP, AFM, fluorescent lifetime imaging, two-photon excitation, optical traps, and interference reflection. Some combinations of these techniques will be discussed later.

\section{Polarized TIRF}

The fluorophores can be excited only if their dipole is parallel to the excitation light dipole, which is called photoselection. A variation of TIRF called polarized TIRF uses polarized light perpendicular to the incidence plane (p-polarized) and parallel to the incidence plane (s-polarized) to overcome this limit. If the dipole of a fluorophore is always parallel to the membrane surface, p-polarized light can help to excite only the regions where the membrane is not parallel to the surface. The investigation of membrane curvature can be an important application field for polarized TIRF (Axelrod 2008).

\section{TIRF Applications on Membrane Dynamics}

There have been many studies to elucidate membrane dynamics using TIRF. Recently, it has been shown that TIRF has the capacity to show the adsorption of proteins and peptides to lipids in SLBs (Fox et al. 2009; Jorgensen et al. 2009). TIRF was combined with singleparticle tracking to show the enrichment of GPI-anchored proteins in sphingolipid rich regions, as proposed by lipid raft theory (Pinaud et al. 2009). A new method has also been applied to detect lipid rafts, called LGTIRF (Sohn et al. 2010). Other applications were to elucidate the role of ceramide in membrane restructuring (Ira et al. 2009), the organization of bacterial light harvesting complex 2 (Dewa et al. 2006), the role of cholesterol in antibody binding (Yu et al. 2009), EGFR activation by EGF (Sako et al. 2000; Cannon et al. 2005; Teramura et al. 2006), and the phase preference of peptides (Choucair et al. 2007). Membrane curvature, exocytosis, and endocytosis are some other topics in which TIRF is successfully applied (Merrifield et al. 2002, 2005; Byrne et al. 2008; Nagamatsu and Ohara-Imaizumi 2008; Joselevitch and Zenisek 2009; Ohara-Imaizume et al. 2009; Aoki et al. 2010; Gorg et al. 2010; Lam et al. 2010).

\section{Two-Photon Microscopy}

The theoretical basis of two-photon excitation was laid in a study of the early 1930s (Goppert 1929), although the experimental realization took almost three decades (Kaiser and Garrett 1961). It was first used in LSM in the 1970s (Hellwarth and Christensen 1975) but a convincing two-photon excitation fluorescence microscopy was only demonstrated in 1990 (Denk et al. 1990).

Two-photon microscopy, as the name implies, uses simultaneous absorption of two longer wavelength photons (at $\lambda_{1}$ and $\lambda_{2}$ ) to excite a fluorophore, which would be usually 
excited by a single photon at a shorter wavelength $\left(\lambda_{3}\right)$. The relationship between the wavelengths is

$$
\lambda_{3}=\frac{1}{\lambda_{1}^{-1}+\lambda_{2}^{-1}} .
$$

Because the two photons have to be absorbed simultaneously to excite the fluorophore, the excitation is dependent on the square of the light intensity. This could be thought as an equivalent of the double selection in confocal imaging, achieved by a selective illumination by the light source and selective detection by a pinhole. Therefore, in the two-photon illumination mode, a pinhole is no longer necessary. Moreover, it minimizes the out-of-focus photobleaching because the excitation only occurs in the vicinity of the focal plane (Fig. 2). Scattering is greatly reduced with two-photon excitation, and penetration depths for the long wavelength excitation are increased.

Because the emission does not have to pass through a pinhole, area detectors can be used and no descanning of the beam is necessary, making detection quite simple.

Another advantage of two-photon microscopy is its ability to excite fluorophores absorbing in the UV by two photons in visible range, which surpasses usual UV transmission problems with glass lenses. In combination with the reduced out-of-focus fluorescence, it also provides a suitable tool for UV uncaging in vivo without significant photo damage.
The photon density in two-photon excitation should be about one million times higher than is required for single-photon excitation, because of the square dependence of the absorption on intensity. Therefore, pulsed lasers should be used with sufficient photon flux in the pulses while having fairly low average power. Titanium-sapphire lasers are extensively used for two-photon microscopes because they provide a wide range of excitation wavelengths between 700 and $1100 \mathrm{~nm}$. Because of different photophysical selection rules, two-photon absorption spectra are not identical with twice the spectra for one-photon excitation, and have therefore to be independently determined. In the same way as for TIR illumination, twophoton excitation can be combined with other single-molecule techniques.

\section{Applications}

Two-photon microscopy is very suitable to excite photosensitive, easily bleachable lipid probes in the blue to near-UV spectral range, such as Laurdan or C-Laurdan. These probes were used to detect the membrane domains in model membranes, as well as in living cells, by two-photon microscopy (Parasassi et al. 1997; Bagatolli and Gratton 1999, 2000a,b; Dietrich et al. 2001; Bagatolli 2003; Bagatolli et al. 2003; Kim et al. 2007; Kaiser et al. 2009). The order of different membrane systems was investigated (Gasecka et al. 2009), and new probes

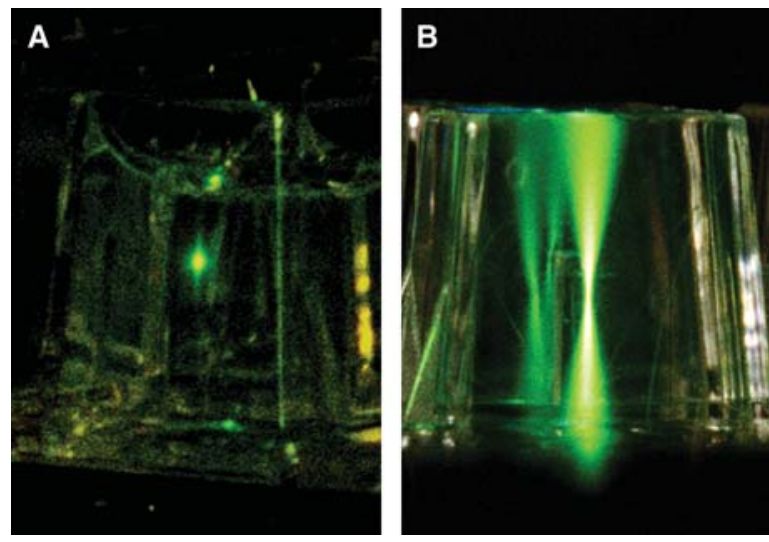

Figure 2. (A) Two-photon illumination and (B) single-photon illumination. 
to visualize the membrane order were tested by two-photon microscopy (Jin et al. 2006; Kim et al. 2008; Klymchenko et al. 2009).

Fluorescence Recovery after Photobleaching (FRAP)

FRAP or, under its previous name, fluorescence photobleaching recovery (FPR) was first described in the late 1970s (Axelrod et al. 1976; Koppel et al. 1976) and got very popular in the 1990s because of the improvements in optics and the discovery of fluorescent proteins.

FRAP is a technique that exploits the photobleaching property of fluorophores. A region of interest (ROI) is bleached with a high laser power. Then, the ROI is observed for fluorescence recovery, caused by diffusion, interactions or reactions of the surrounding fluorophores, which yields a recovery curve. This curve typically looks like the one shown in Figure 3. Its most remarkable features are the bleaching step, an exponential-like recovery with characteristic half-time, and a recovery level usually lower than the initial level, whose offset is the so-called "immobile fraction."

According to the steepness of the recovery, diffusion coefficients, binding rates or turnover rates can be determined. The steeper the recovery is, the faster the molecules are, with diffusion

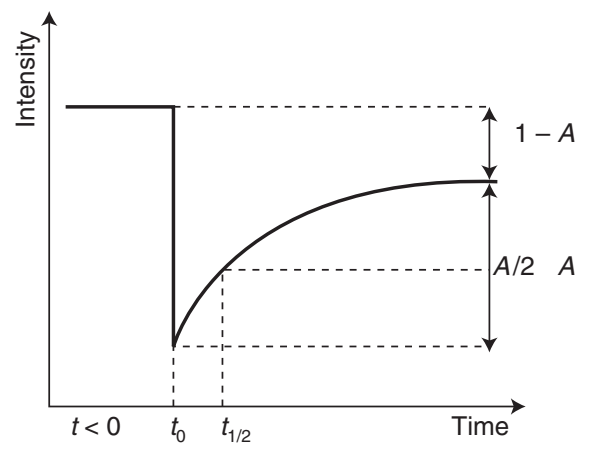

Figure 3. FRAP parameters. $0<t$ is the time before the bleaching, $t_{0}$ is the time at which bleaching occurs, $t_{1 / 2}$ is the half-life (i.e., the time at $A / 2$ ), $A$ is the mobile fraction, and $1-A$ is the immobile fraction. coefficients $(D)$ determined by the Stokes-Einstein relationship for spherical molecules:

$$
D=\frac{k_{\mathrm{B}} T}{6 \pi \eta r},
$$

where $k_{\mathrm{B}}$ is the Boltzman constant, $T$ is the absolute temperature, $\eta$ is the viscosity, and $r$ is the hydrodynamic radius of the spherical particles.

Because photobleaching is an irreversible process, immobile molecules will not recover at all. Therefore, one can obtain the immobile fraction of molecules as an additional information from FRAP curves. To get accurate quantitative information, the intensity profiles of bleached ROI, as well as an unbleached positive reference ROI on the same membrane, an empty ROI outside of the membrane, and the whole cell or membrane have to be derived. Then, the recovery should be normalized according to these values (Kenworthy 2007).

Normalized FRAP curves should be fitted to the appropriate models, to get the half time which is the time required for half of the recovery. The simplest fitting is by a single exponential:

$$
f(t)=A\left(1-e^{-\tau t}\right) .
$$

The diffusion coefficient can then be determined as

$$
D=\frac{r_{0}^{2} \gamma}{4 \tau_{1 / 2}},
$$

where $r_{0}$ is $1 / e^{2}$ radius of the Gaussian laser beam, $\gamma$ is the parameter that depends on the photobleaching extend varying from 1.0 to 1.2 depending on ROI shape, and $\tau_{1 / 2}$ is the halftime. In case of a uniform circular spot ROI,

$$
D=0.224 r^{2} / t_{\mathrm{d}} \text {. }
$$

Many fitting models for diffusion, interaction and reaction have been developed recently, some of which are listed in Table 1.

\section{TIR-FRAP}

To measure the diffusion of molecules in a membrane, TIR illumination can be a better option than confocal microscopy for the same 
Fluorescence Techniques to Study Lipid Dynamics

Table 1. FRAP fitting models

\begin{tabular}{|c|c|c|}
\hline Type of model & Function & Reference \\
\hline Diffusion & $f(t)=f_{f}\left(1-\left(\frac{w^{2}}{w^{2}+4 \pi D t}\right)^{1 / 2}\right)$ & Ellenberg et al. 1997 \\
\hline Diffusion & $f(t)=e^{-\frac{\tau_{D}}{2 t}}\left[I_{0}\left(\frac{\tau_{D}}{2 t}\right)+I_{1}\left(\frac{\tau_{D}}{2 t}\right)\right]$ & Soumpasis 1983 \\
\hline $\begin{array}{l}\text { Chemical interaction } \\
\text { dominant }\end{array}$ & $f(t)=y_{0}+A e^{\tau_{1} t}$ & Phair et al. 2004 \\
\hline Reaction dominant & $f(t)=1-C_{\mathrm{eq}} e^{-k_{\mathrm{off}} t}$ & Sprague et al. 2004 \\
\hline
\end{tabular}

reasons as listed above. With TIR-FCS, complex binding-unbinding measurements are possible with high accuracy because of well-defined and background free illumination.

\section{Challenges and Artifacts in FRAP}

FRAP is usually implemented in laser scanning confocal microscopes (LSCMs), thus its ability is limited by the features of the respective microscope. In FRAP experiments, it is usually assumed that all the molecules are bleached at the same time, and no diffusion happens during photobleaching. But both assumptions may be wrong at nonideal settings in an LSCM. To guarantee proper photobleaching, more than one scan at high laser power may be needed. However, as the number of scanning cycles increases, diffusion into the ROI is nonnegligible, especially for fast diffusing molecules. This leads to a wrong initial starting point of recovery, and yields a wider and shallower bleaching profile. In other words, the required time for molecules to recover the bleached area appears to be higher. To get rid of this artifact, the initial point needs to be calibrated carefully (Snapp et al. 2003; Weiss 2004).

ROI Size. The shape and the area of the FRAP ROI are crucial for the extraction of diffusion coefficients. The ROI size should be much smaller than the total size of the sample, to keep the overall fraction of photobleached molecules fairly low, not to influence the fluorescence intensity profile of the sample. Moreover, ROI radius should not exceed $1 \mu \mathrm{m}$ for Gaussian approximation to be valid.
Photobleaching Artifacts. During photobleaching, many chemical reactions can happen because of the high laser power, induced by radical (often reactive oxygen) formation, such as protein cross linking. This may affect the concentrations but also the diffusion coefficient notably. Another effect of high laser power, particularly in the red spectral range, can be a slight local temperature rise during photobleaching. Although it has been shown that the temperature increase is minor (Axelrod 1977) in solution, it may be important on membranes, specifically at critical temperature points (Honekamp-Smith et al. 2008).

Besides experimental parameters mentioned above, a proper fitting should be carefully applied (Sprague and McNally 2005).

\section{Applications}

The diffusion in native cell membranes has been addressed using the FRAP technique since long (Lippincott-Schwartz et al. 2001, 2003). The first studies were performed to see whether membrane heterogeneity affects the diffusion of proteins in the membrane (Edidin 1992; Jacobson et al. 1995; Feder et al. 1996; Lommerse et al. 2004; Kenworthy 2005; Lagerholm et al. 2005), which resulted in anomalous diffusion concept. In the context of lipid rafts, a continuous effort has been made to distinguish between the diffusion of raft and nonraft markers, as well as to characterize the factors that can influence the membrane organization, like cholesterol (Niv et al. 1999, 2002; Hao et al. 2001; Kenworthy et al. 2004; Rotblat et al. 2004; Goodwin et al. 2005; Roy et al. 2005; Meder et al. 2006; Nicolau 
et al. 2006; Shvartsman et al. 2006). TIR-FRAP was applied to calculate the rates of binding and unbinding of hormones to and from the cell surface (Hellen and Axelrod 1991; Fulbright and Axelrod 1993).

\section{Fluorescence Correlation Spectroscopy (FCS)}

FCS is a method that has been extensively used and further developed by our group, being introduced and established as a very suitable approach to characterize model and cellular membranes (Schwille et al. 1999a; Bacia et al. 2004). It is, in a way, a single-molecule method, but provides sufficient statistical significance to also use it for general characterization of membranes, mainly through the diffusion properties of their constituents. It is thus related to FRAP, but provides several advantages, the most crucial of which is the dramatically improved sensitivity, allowing to work at significantly reduced fluorescence labeling densities. FCS has long been used to characterize domainforming membranes (Korlach et al. 1999), and recently, by combination with super resolution illumination (Eggeling et al. 2009), was able to resolve nanometer-sized entrapment sites of labeled raft-markers. Because of our intensive efforts on FCS applied to membranes, this technique will be discussed in more detail in the following.

FCS measures small fluorescence intensity fluctuations in a defined volume. It provides accurate information about diffusion coefficients, concentrations, molecular brightness, intramolecular dynamics, and molecular interactions. It has been extensively used for a variety of biological applications, because of its great sensitivity. FCS has been combined with many different imaging methods, such as laser scanning confocal microscopy, two-photon microscopy, total internal reflection fluorescence microscopy, stimulated emission depletion nanoscopy, and others, making it particularly feasible for cell biology.

FCS was first established in the 1970s (Magde et al. 1972, 1974, 1978; Elson and Magde 1974) and technically greatly improved in the following years (Rigler et al. 1993; Eigen and Rigler 1994). Fluorescence intensity fluctuations, primarily addressed by FCS, can be caused by diffusion of the molecules through the observation volume, or by reversible brightness changes of the molecules because of some chemical or photophysical reactions (Petrov and Schwille 2007). FCS performs the statistical analysis of these fluctuations. In other words, it correlates a signal at a certain time $t$ with the same signal after a lag time $t+\tau$, and takes the temporal average. This correlation can be described as self-similarity of the signal in time, which is represented by the autocorrelation function, a temporal decay function of average fluctuations. The basic formula for the fluctuation autocorrelation function is

$$
G(\tau)=\frac{\langle\delta F(t) \cdot \delta F(t+\tau)\rangle}{\langle F(t)\rangle^{2}},
$$

where $\delta F(t)=F(t)-\langle F(t)\rangle$ is the fluctuation around the average intensity and \langle\rangle denotes the temporal average; $\tau$ is the lag time. The denominator is for normalization.

The basic steps of FCS experiments are as follows. First, the sample is illuminated by the appropriate illumination technique. Generally, in the simplest representation of FCS, confocal illumination without beam-scanning is used. The fluorescence signal is collected by the objective and detected by sensitive photodetectors, often by avalanche photodiodes (APDs). After detecting the fluorescence intensity for a certain time, a hardware correlator usually correlates the signal from subsequent time points according to the correlation function mentioned above, and forms the experimental FCS curve. This correlation step can also be performed retrospectively, if data is recorded in small enough $(<\mu \mathrm{sec})$ time bins. Then, the correlation curve as in Equation 16 is fitted by an appropriate fitting model (some listed below) to get the numerical values of diffusion times, concentrations and molecular brightness, or other parameters governing fluctuation decay.

As seen in Figure 4, the amplitude of the curve is reciprocal to the concentration. The reason behind this is that for lag time zero, 

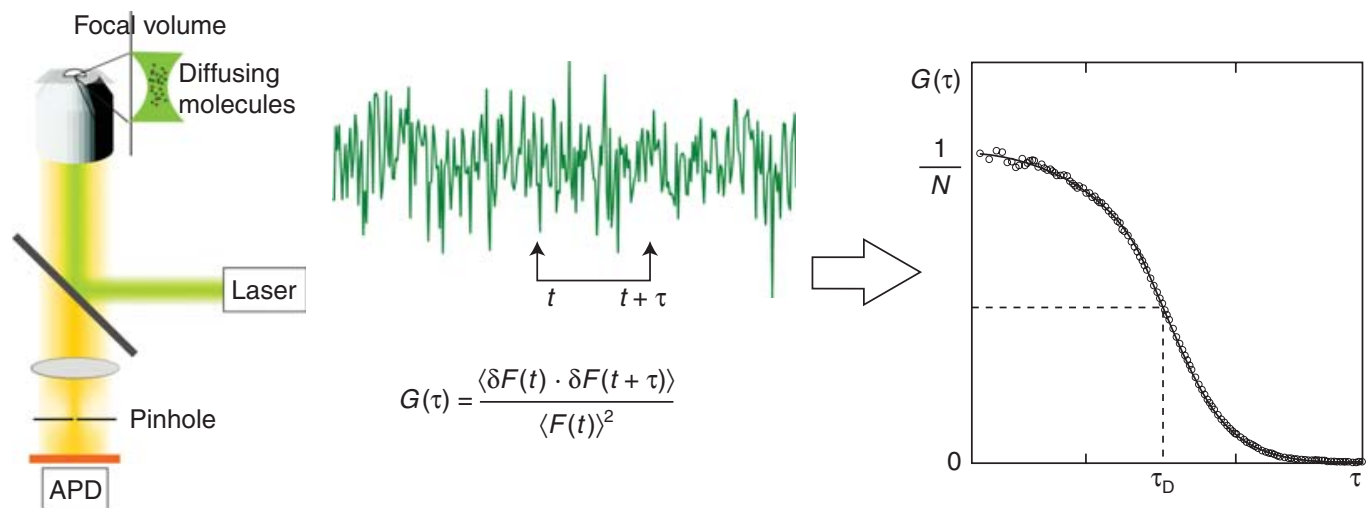

Figure 4. Principle of FCS. (Image courtesy of Jonas Ries.)

$\mathrm{G}(\tau)$ is determined as

$$
G(0)=\frac{\left\langle(\delta F)^{2}\right\rangle}{\langle F\rangle^{2}} .
$$

For random processes that are governed by Poisson statistics, the variance is

$$
\operatorname{Var}(N)=\left\langle(\delta N)^{2}\right\rangle=N .
$$

Because the fluorescence intensity is directly proportional to the number of molecules,

$$
\langle F\rangle=q\langle N\rangle,
$$

when the intensity is normalized, we get

$$
\frac{\left\langle(\delta F)^{2}\right\rangle}{\langle F\rangle^{2}}=\frac{\left\langle(\delta N)^{2}\right\rangle}{\langle N\rangle^{2}}=\frac{\langle N\rangle}{\langle N\rangle^{2}}=\frac{1}{\langle N\rangle} .
$$

As stated above, the diffusion time and other variables are obtained from fitting the experimental data to the proper model function. From the diffusion time $\tau_{\mathrm{D}}$, the diffusion coefficient can be determined if the diameter of the focal volume is known:

$$
\tau_{\mathrm{D}}=\frac{\omega_{\mathrm{o}}^{2}}{4 D}
$$

Here, $\omega_{\mathrm{o}}$ is the beam waist of focal volume (i.e., the radial distance of the optical axis), and $D$ is the diffusion coefficient.

Besides the concentration and the diffusion time, the brightness of the molecule, $\eta$, can be calculated. This parameter is quite important for a good statistical accuracy, and can be used to assess the quality of FCS measurements in general. However, it can also reflect on the formation of higher molecular complexes and aggregates. $\eta$ is directly proportional to the total photon count and to the amplitude of the correlation function:

$$
\eta=\frac{\langle F(t)\rangle}{N}=\langle F(t)\rangle \cdot G(0) .
$$

In practice, FCS is quite a complicated and delicate technique to apply, with many parameters that have to be taken into account and carefully controlled.

- If the concentration of the fluorophores is too high $(>100 \mathrm{~nm})$ the contribution of correlated photons to the total intensity (or, the strength of the fluctuations) is only marginal, and precludes their analysis. If the concentration is too low $(<1 \mathrm{pm})$ it gets difficult to register a molecule in the focal volume during a sensible measurement time. Background noise dominates the signal. In both cases, it is difficult to record decent FCS curves.

- Autofluorescence and (scattering) background may always affect the total fluorescence intensity, and there should be elaborate corrections for them. Besides, the sample should be kept in a nonautofluorescent medium. 
- Low laser power should always be used to avoid photobleaching.

- The acquisition time should be long enough to collect enough photons to correlate, but not too long to avoid photobleaching.

- Fluorophore selection should be made carefully; more than other techniques FCS requires a high photostability.

The basic steps and tricks to do FCS on living cells are well described (Kim et al. 2007).

More problems, precautions, and corrections will be discussed later.

\section{Fluorescence Cross-Correlation Spectroscopy (FCCS)}

To quantitatively characterize molecular interactions is of prime importance for cell and molecular biologists. Biochemical assays that are usually employed for this purpose are particularly problematic for molecules embedded in or attached to cell membranes, because the physiological environment cannot be closely preserved in vitro. Video microscopy on fluorescently labeled molecules in life cells has helped to some extent, but often produces ambiguous results, because it largely relies on simple colocalization that does not really probe interaction, but rather spatial proximity. As the diffraction limit is much larger than a protein size, when two proteins are closer than the resolution of the microscope, it cannot be determined whether they are truly interacting. A much better approach is FRET, relying on the radiation-less transfer of excited state energy between two or more molecules that carry fluorescent labels with large spectral overlap. Here, the proximity needs to be in nanometer range, making it much more specific to probe true binding. FRET will be discussed in detail later. However, the key challenge with FRET is to attach the labels close enough to the binding site to yield high transfer efficiency, but far enough apart not to interfere with the binding. Here, a variant of FCS, called fluorescence crosscorrelation spectroscopy (FCCS) often provides a valuable alternative.
FCS itself provides detailed information on the diffusion properties of labeled molecules. To probe binding or interaction of small molecules to large ones, or to immobile structures such as the cell membrane, the reduction in diffusional mobility may provide valuable information on the binding process. This has in the past been used to characterize binding events by standard one-color FCS (Icenogle and Elson 1983a,b). However, this approach breaks down when the interaction between molecules of approximately the same size are to be analyzed. Simulations show that for the minimum detectable difference in diffusion time of a molecule of at least 1.6-fold, an approximately sixfold change in mass is required, as implicitly seen in Equation 12, where the diffusion time is inversely proportional to the third root of mass (Meseth et al. 1999).

The principle of FCCS is to observe codiffusion of molecules, rather than colocalization. It can thus be employed to probe any phenomenon leading to or terminating such a comovement (Schwille et al. 1997). Direct interaction, complex formation, but also the clustering of molecules in microdomains or nanodomains can lead to such a codiffusion of two molecules of separate species. In contrast to standard FCS, the mathematical routine for FCCS is to correlate the fluorescence fluctuations from the first channel at time $t$ with the fluorescence fluctuations in the second channel at time $t+\tau$ over a certain measurement interval (Fig. 5). The cross-correlation function $G_{\mathrm{cc}}(\tau)$ for FCCS is given by

$$
G_{\mathrm{cc}}(\tau)=\frac{\left\langle\delta F_{1}(t) \cdot \delta F_{2}(t+\tau)\right\rangle}{\left\langle F_{1}(t)\right\rangle\left\langle F_{2}(t)\right\rangle},
$$

where $\delta F_{1}(t)$ and $\delta F_{2}(t)$ are the fluctuations in the two fluorescence signals, and $\left\langle F_{1}(t)\right\rangle$ and $\left\langle F_{2}(t)\right\rangle$ are the mean intensities.

\section{Scanning FCS (sFCS)}

Although FCS is still mostly performed with a steady measurement volume (i.e., a confocal spot parked at a fixed position in solution or in a cell), many modern instruments, particularly combined FCS-LSM modules, feature 

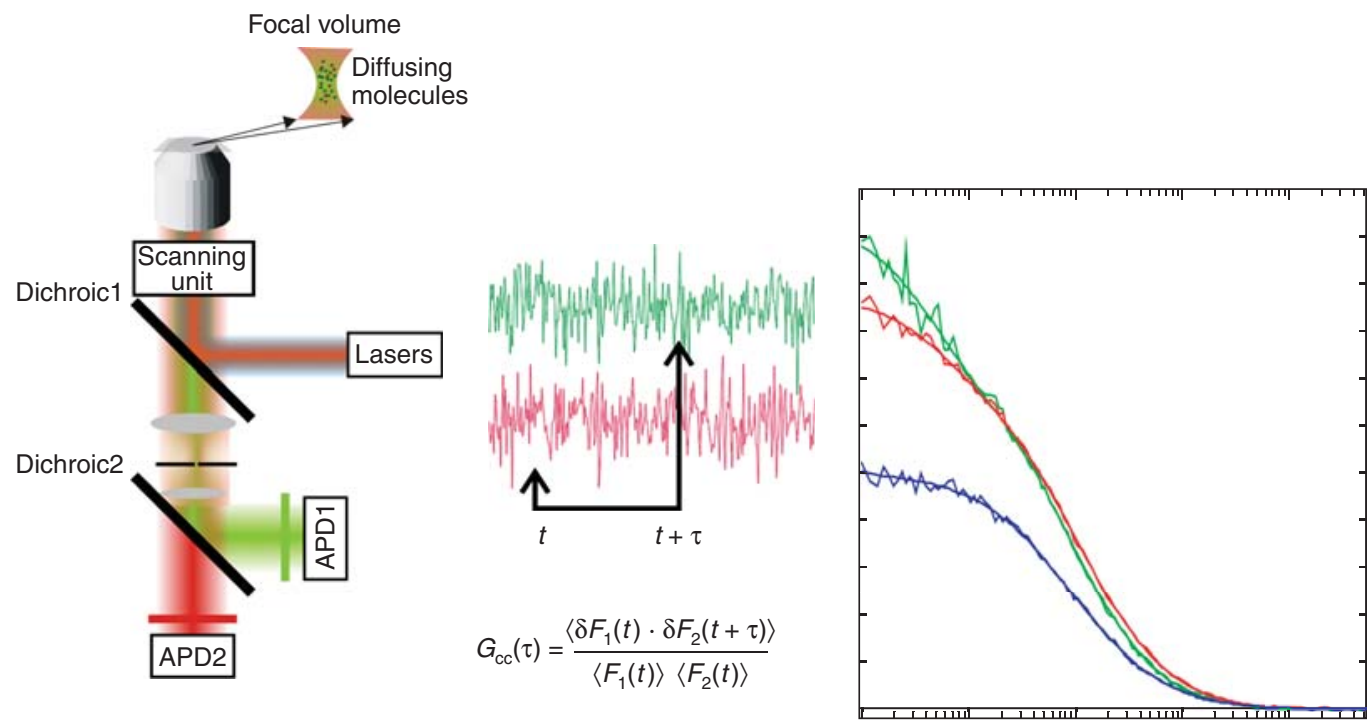

Figure 5. Principle of FCCS. (Image courtesy of Jonas Ries.)

the combination of correlation analysis with a scan beam. Here, the focal volume may be moving on a line (Petersen 1986; Petersen et al. 1986), a circle in lateral axis (Petrasek and Schwille 2008; Petrasek et al. 2010) or along $z$-axis (Benda et al. 2003). The basic principle of scanning FCS perpendicular to the membrane is shown in Figure 6.

In the line-scan mode, the laser focus moves perpendicularly to an axially aligned membrane (e.g., the side membrane of a giant vesicle).
Because of the scan path being much larger than the actual intersection with the membrane, the focal spot will only be illuminating (membrane-bound) molecules for a small fraction of time. Thus, the recorded time-intensity trace cannot be directly fed into the hardware correlator but has to be processed by software to align the actual membrane intersections, before actually correlating the processed pseudoreal time signal and fitting the data (Ries and Schwille 2006). This procedure, although more

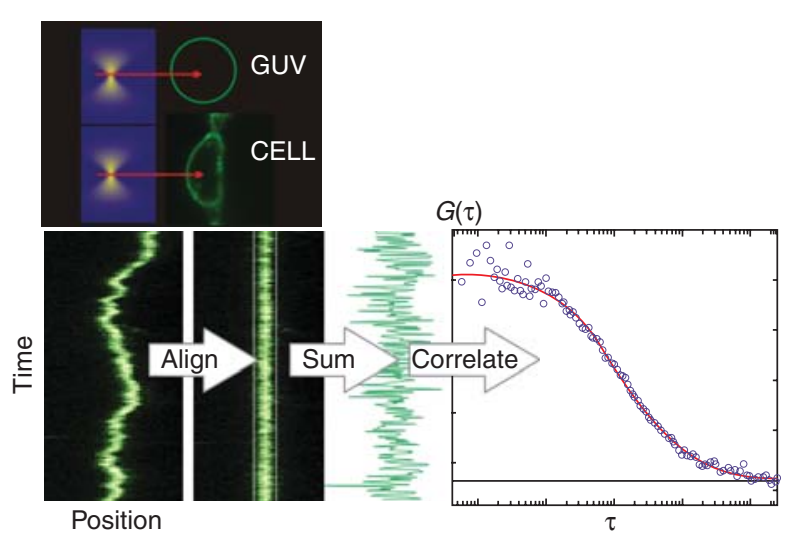

Figure 6. Principle of sFCS. (Image courtesy of Jonas Ries.) 
elaborate than standard FCS, has the enormous advantage that sample drift or large-scale signal disturbance by autofluorescent particles can be efficiently suppressed. Although line-scan FCS has many advantages for membrane systems, which are going to be discussed later, it may cause out of focus bleaching, and its time resolution is limited by the software processing, rendering it unattractive to detect fast diffusing components in solution.

Besides a line scan in the lateral direction, there is an axial scanning approach in which the focal volume moves in the $z$ direction, so that it can measure above the membrane, at the membrane and below the membrane sequentially (Humpolickova et al. 2006). Because of the laser divergence, the size of the illuminated area above and below the focal plane are larger, rendering the number of molecules and the diffusion time higher when moving the focal spot away from the focal plane. Also, the movement is usually performed by stage scanning, with much lower scan speed than available for lateral scanning, Consequently, this $z$ scan is usually performed for other purposes: for example, to calibrate the focal volume in the $z$ direction or to vary the size of the illuminated area on the membrane with minimal efforts.

\section{Two-Focus FCS}

Another modification of FCS that bears the characteristics of cross-correlation is two-focus FCS (or dual focus FCS). It can be implemented with two fixed confocal volume elements displaced with respect to each other at a spatial distance. This setup simplifies calibration-less measurements of diffusion coefficients (Dertinger et al. 2007). Spatial cross-correlation with two focal elements can, however, be conveniently combined with line scanning, in which two identical lines at a known distance $d$ are scanned by two foci simultaneously, or alternately with very high frequency. When the distance is well known, one can extract autocorrelation curves as well as the spatial cross correlation curve between two foci. This mode is quite insensitive to artifacts that originate from the variations of the focal volume (e.g., because of different refractive indices within the sample [Dertinger et al. 2007; Loman et al. 2008]), and therefore particularly suited for cellular FCS. This will be discussed later.

\section{TIR-FCS}

As mentioned before, TIR illumination provides a great axial resolution, which makes it a very appropriate tool for membrane research. It can also be conveniently combined with FCS on membranes or surfaces in general. If there is a strong background from labeled molecules in the cytosol, or above and below the surface of interest, selective data processing in scanning FCS or two-focus FCS can only partly overcome this problem, which usually leads to a decrease in amplitude in the correlation curve. As a much more elegant strategy to eliminate the background caused by any other molecules away from the surface, objective-type TIRF illumination combined with standard confocal detection can be applied (Schwille 2003; Ries et al. 2008).

\section{Two-Photon FCS}

To combine FCS with two-photon excitation provides a number of interesting features and advantages. First of all, like for standard imaging, it limits cumulative photobleaching in out-offocus areas, making it preferable for FCS measurements on samples with limited dye resources such as small cells and organelles (Schwille and Heinze 2001; Schwille et al. 2009). Two-photon excitation is, further, the method of choice for samples of high turbidity or high scattering cross sections, like multicellular systems or cells with thick cell walls. Additional advantages may be provided by the photophysical properties of the dyes, allowing to coexcite and correlate up to three spectrally distinct fluorophores with one two-photon excitation beam (Heinze et al. 2000, 2002). Caution has to be applied with regard to the photostability of dyes and the available count rate per molecule, as both seem to be significantly reduced under two-photon excitation (Schwille et al. 1999b). 


\section{Difficulties and Artifacts in FCS Applications}

Background. Background can be caused by scattering, autofluorescence, or unwanted fluorophores in the sample because of nonspecific labeling. If the background is truly random and noncorrelated, a signal can be easily background-corrected after the measurement as follows (Petrasek et al. 2010):

$$
g_{c}(\tau)=\left(\frac{\langle F\rangle}{\langle F\rangle-B}\right)^{2} g(\tau)
$$

where $g_{c}(\tau)$ is corrected nonnormalized correlation, $g(\tau)$ is measured nonnormalized correlation, $B$ is background fluorescence measured on the sample without fluorescent molecules, and $\langle F\rangle$ is the average intensity.

In case of correlating background, it has to be added to the fitting functions as a fixed second component in a two-component model after being carefully calibrated.

Membrane Heterogeneities, Blinking, Triplet, and Photobleaching. FCS usually assumes an equilibrated steady state in the focal volume around which fluctuations occur randomly. This means that the average count rate should not change over time when recording an FCS curve. In reality, this assumption is very rarely true. Many events, above all cumulative photobleaching, cause a continuous drop or other large scale drift in the average count rate,
Fluorescence Techniques to Study Lipid Dynamics

rendering the error-free recording of FCS curves quite complicated.

In measurements on live cell membranes, membrane undulations constitute the main problem. As the membrane may always be moving on a micron scale, the fraction of membrane occupying the detection volume can change drastically. This causes an increase or decrease in count rate, and results in major distortions of the curve, leading to erroneous values for autocorrelation amplitude and diffusion time.

Another cause of severe, although better controllable, distortion of FCS curves is the photophysical phenomenon of triplet state population, in which the molecules are trapped in a dark state for a few microseconds. Tripletinduced photophysical dynamics may lead to wrong fitting of diffusion times, particularly if the triplet fraction is high and the diffusion times are short (Davis and Shen 2006). Yet, this phenomenon can usually be corrected for in the fitting function, as seen in Table 2. Usually, triple dynamics can be easily distinguished from diffusion because it is independent of volume size, but dependent on illumination power. It can be easily evidenced as an additional shoulder in the FCS curve on short timescales.

Blinking on short timescales does not have to be of photophysical origin. Several fluorophores, particularly fluorescent proteins such as GFP, exhibit excitation independent dark statebright state transitions which may, however,

Table 2. FCS fitting models

\begin{tabular}{ll}
\hline Diffusion type & \multicolumn{1}{c}{ Fitting function } \\
\hline 3D diffusion & $G(\tau)=\frac{1}{N}\left(1+\frac{\tau}{\tau_{\mathrm{D}}}\right)^{-1} \frac{1}{\sqrt{1+w_{0}^{2} \frac{\tau}{\mathrm{T}}}}$ \\
2D diffusion & $G(\tau)=\frac{1}{N}\left(1+\frac{\tau}{\tau_{\mathrm{D}}}\right)^{-1}$ \\
2D diffusion for elliptical Gaussian profile & $G(\tau)=\frac{1}{N}\left(1+\frac{\tau}{\tau_{\mathrm{D}}}\right)^{-1 / 2} \frac{1}{\sqrt{1+\frac{\tau}{\mathcal{S}^{2} \mathrm{D}}}}$ \\
2D diffusion with triplet & $G_{\mathrm{Tr}}(\tau)=G(\tau)\left[1+T(1-T)^{-1} \exp \left(\frac{-\tau}{\tau_{\mathrm{Tr}}}\right)\right]$ \\
2D diffusion with blinking & $G_{\mathrm{B}}(\tau)=G(\tau)\left[1+\frac{C_{\mathrm{dark}}}{C_{\mathrm{bright}}} e^{-k_{\mathrm{bl}} \tau}\right]$ \\
2D diffusion with two-component & $G_{2 \mathrm{C}}(\tau)=\frac{1}{N_{\mathrm{tot}}} \frac{q_{1}^{2} Y_{1} G_{1}(\tau)+q_{2}^{2} Y_{2} G_{2}(\tau)}{q_{1} Y_{1}+q_{2} Y_{2}}$ \\
\hline
\end{tabular}


be dependent on $\mathrm{pH}$ or ionic strength of the solution (Haupts et al. 1998). After careful calibration, this can be incorporated into the fitting function in the same way as the triplet dynamics (Table 2).

A more severe problem for FCS is dye photobleaching, as it not only leads to signal loss, but also compromises the determination of concentrations and diffusion coefficients, the key parameters in FCS applications. At too high illumination intensities, molecules will not stay fluorescent during their full diffusion path through the detection volume, but will be destroyed before leaving it again, leading to erroneously low diffusion times, and an overestimation of diffusion coefficients. In living cells, there is an additional problem with immobile fluorophores being unavoidably bleached during the measurements and leading to a drift in the overall count rate. To prevent this effect from destroying the correlation curve during real-time recordings, a prebleaching is usually performed. Although photobleaching can be usually diagnosed by decay in count rate, its effect is not necessarily visible on first sight, because an equilibrium state may be reached between bleaching and continuous supply of new fluorophores. To rule out photobleachinginduced artifacts in general, a laser power series of FCS measurements from minimum to a moderate power should be recorded. Only for power levels that do not show a change in the curve parameters, compared to very low power measurements, the intensity can be assumed safe (Dittrich and Schwille 2001; Delon et al. 2004). This "safe intensity" is, however, dramatically dependent on the diffusion characteristics of the labeled molecules (lower intensities required for slower molecules), and cannot just be inferred from pure dye measurements.

For measurements on extremely slow particles, scanning FCS as explained above represents an efficient solution to avoid photobleachinginduced artifacts, because the laser is not continuously exciting the same spot, reducing the interaction time with a specific region.

Detector Dead Time and Saturation. When a photon hits the APD detector, it creates an avalanche of electrons to amplify the signal. Before the next photon can be registered, there is a short interval of $\sim 100 \mathrm{nsec}$, called the dead time of the detector. Events occurring on shorter scales than the dead time cannot be resolved. Sometimes, the detection of a single photon triggers the APD chip to create a second cascade during the dead time, the so-called "afterpulsing," which is an artifactual event, but highly correlated with the first one. As a result, a peak in the correlation curve is observed at very short timescales. The simplest solution for this (usually hardware-induced) problem is to split the light into two detection channels and record the cross-correlation between them. Cross-correlation does not include this after-pulsing peak because it is a hardwareinduced phenomenon in only one of the detectors.

There is a photon count limit for the detector that it can process at a time. Above this value, electronic saturation occurs, which has a similar effect as optical saturation in the sample. Optical saturation happens when most of the molecules in the focal volume are not in the ground state, instead in excited state or triplet state. This effect usually leads to an enlarged focal volume and results in a slower decay of the correlation function (Gregor et al. 2005; Humpolickova et al. 2009). It should be ruled out in the same way as for photobleaching, by recording a laser power series and staying well below the intensity at which the curves change their shape.

Focal Volume Geometry and Positioning. The probe volume (composed of illumination by the laser and detection via the pinhole) is usually approximated as a 3D Gaussian profile. In one photon excitation, slightly underfilling the back aperture of the objective is a good way to satisfy this approximation. Overfilling the aperture to yield better excitation efficiencies, on the other hand, will for one-photon excitation result in diffraction fringes of the back aperture itself. This non-Gaussian volume is prone to produce artifacts in diffusion time, which may be misunderstood and taken as a second species or kinetics (Hess and Webb 2002). 
There are several additional factors, like optical aberrations, that distort the geometry (shape or size) of the focal volume from the Gaussian profile.

Refractive index mismatch could be a common problem when dealing with cells, in which refractive indices vary from 1.33 to 1.38. When there is a mismatch between the immersion liquid, glass, and the sample, aberrations occur which cause a larger detection volume than assumed by the fitting model. This results in larger diffusion times and lower diffusion coefficients than the real values. Similar to this, displacement of the pinhole along the optical axis leads to larger detection volumes and larger diffusion times. Coverslip thickness also affects the focal volume. Objectives are usually designed for a certain range of coverslip thickness that has to be adjusted exactly. Deviations from the correct value again result in a larger detection area and underestimated diffusion coefficients (Enderlein et al. 2004, 2005).

Artifacts caused by refractive index mismatch, pinhole misalignment, or coverslip thickness affect the control experiments in the same way as the measurements, such that the ratio of control over sample is still correct. If absolute values are to be obtained and the optical system cannot be easily corrected, twofocus FCS provides a good solution to avoid problems with detection volume deformations. Two-focus FCS is insensitive to refractive index mismatch, cover-slide thickness variation, and optical saturation. Therefore, it happens to be a focal volume-calibration free technique for accurate dynamics measurements (Dertinger et al. 2007; Loman et al. 2008).

Correct axial positioning of the detection volume is crucial for membrane analysis. If the center of the focal volume is not exactly on the membrane, the divergent laser beam illuminates a bigger area of the membrane, mimicking a higher concentration (through the reduced count rate and higher occupation number) and a smaller diffusion coefficient. To minimize this artifact, the count rate should be maximized when adjusting the $z$ position. For a more decisive solution, positioning-calibration
Fluorescence Techniques to Study Lipid Dynamics

free FCS variants like scanning FCS or $z$-scan FCS should be used.

Specific Artifacts in FCCS. One of the most crucial tasks for dual-color cross-correlation is the careful determination of measurement volumes. Because of the different wavelengths, the Airy disc sizes for the two detection channels vary in proportion to their wavelength. Consequently, in most FCCS instruments, the focal volumes do usually not completely overlap, even after eliminating all aberrations (Weidemann et al. 2002). For quantitative FCCS, this requires intensive calibration measurements (Schwille et al. 1997) (e.g., by using a "gold standard" of up to $100 \%$ cross-correlation [like a strong receptor-ligand or dsDNA] and comparing the experimental results with this reference).

One of the biggest problems in most optical systems featuring multicolor applications is spectral cross talk. FCCS is particularly prone to producing false positive results because of the cross talk induced by the leakage of the green dyes's emission into the red dyes' detection channel. In that case, the autocorrelation between the red and green spectral parts of the green dye results in false positive cross correlation.

As for other artifacts, cross talk can be taken care of by careful calibration. The cross talk coefficient of any fluorophore $\kappa_{x}$ can be easily calculated by measuring the fluorescence simultaneously in both channels. This coefficient is specific for a particular set of optics (dichroics, filters, etc.):

$$
\kappa_{x}=F_{\mathrm{r}} / F_{\mathrm{g}},
$$

where $F_{\mathrm{r}}$ is the fluorescence intensity in the red channel, and $F_{\mathrm{g}}$ is the fluorescence intensity of the same fluorophore in the green channel.

Cross-correlation can be corrected according to this coefficient in measurements with two fluorophores:

$$
G_{\mathrm{CC}}(\tau)=\frac{F_{\mathrm{g}} F_{\mathrm{r}} G_{\mathrm{rg}}(\tau)-\kappa_{x} F_{\mathrm{g}}^{2} G_{\mathrm{g}}(\tau)}{F_{\mathrm{g}}\left(F_{\mathrm{r}}-\kappa_{x} F_{\mathrm{g}}\right)} .
$$

In cases where cross talk constitutes a substantial portion of the fluorescence signal in the red channel, as is the case for most 
combinations using fluorescent proteins (because of the limited availability of far redemitting FPs), it may be more appropriate to eliminate cross talk already in the measurements, rather than correcting for it retrospectively. Here, alternating excitation schemes have proven to be very powerful. The best known scheme for FCS is pulsed interleaved excitation (Mueller et al. 2005; Sohn et al. 2010), and alternating excitation may also easily be combined with scanning FCS (Ries et al. 2009a).

\section{FCS Applications on Membrane Dynamics}

Over the last decade, FCS has been established as an extremely attractive tool for in vivo (Mütze et al. 2009) studies and on model membrane systems (Kahya and Schwille 2006a). Thus, lipid biology has widely exploited this technique. FCS experiments have been designed and appropriate models have been developed to distinguish free diffusion from diffusion in microdomains and meshwork structures in native membranes (Wawrezinieck et al. 2005; Lenne et al. 2006; Wenger et al. 2007). There have been many studies on phase separated model membranes, supported or free-standing, to determine the diffusion characteristics of lipids in different phases (Chiantia et al. 2008, 2009; Lingwood et al. 2008; García-Sáez and Schwille 2010; García-Sáez et al. 2010). It has been shown that the diffusion coefficient is influenced by environmental conditions such as ionic strength or sugar content of the medium (Bockmann et al. 2003; Sum et al. 2003; Doeven et al. 2005; van den Bogaart et al. 2007; Guo et al. 2008; Vacha et al. 2009). The role of cholesterol in membrane organization, a big issue in lipid biology, has been intensively addressed by FCS (Scherfeld et al. 2003; Bacia et al. 2004, 2005; Kahya and Schwile 2006b). Markers for more ordered lipid environments, such as sphingomyelin and ceramide, were other important molecules to be studied (Chiantia et al. 2007, 2008; Eggeling et al. 2009). Other membrane-dependent processes were also successfully investigated by FCS. For instance, the interaction of morphogen Fgf8 with its receptors on the cell surface in living embryos has been quantitatively determined by using scanning FCCS (Ries et al. 2009a; SR Yu e al. 2009). Another derivative of scanning FCS-line-scan FCS-was developed to address the questions related to membrane dynamics (Ries et al. 2009b). Moreover, reconstituted protein-protein interactions on GUV membranes were monitored by using FCCS. For example, active $\mathrm{tBID}$ and $\mathrm{BCL}_{\mathrm{XL}} \Delta \mathrm{Ct}$ proteins were found to interact, and it was shown that membrane promotes their interaction (Fig. 7) (García-Sáez et al. 2009). In another example, helix-helix interaction was shown for trans-membrane domains using giant plasma membrane spheres by scanning FCCS (Worch et al. 2010).

The applications of FCS in lipid cell biology have been elaborately reviewed elsewhere (Machan and Hof 2010).

\section{A Comparison between FRAP and FCS for Lateral Diffusion}

As seen above, FRAP and FCS are alternative methods to measure lateral diffusion of molecules. Although their specific strengths and shortcomings have been briefly mentioned, a direct comparison may still be helpful for choosing the right technique for a particular experiment.

First, FRAP usually requires higher concentrations than does FCS. Numerically, approximately 100 labeled molecules should be on $1 \mu \mathrm{m}^{2}$ to obtain a reliable FRAP curve (Wolf 1989), and with increasing concentrations, the signal-noise ratio can be improved. In contrast, the FCS curve deteriorates with increasing concentration. One labeled molecule in the detection volume of $0.5 \mathrm{fL}$ (which is almost 20 times less than probed by FRAP) is usually sufficient to record a decent FCS curve. FCS has a much better temporal resolution down to submicroseconds, thus can resolve very fast diffusions (Gordon et al. 1995). On the other hand, FCS is not well suited to analyze slow diffusion, which is quite vulnerable to photobleaching. The high temporal resolution makes FCS also much more susceptible to sample-induced noise, such as membrane 
A

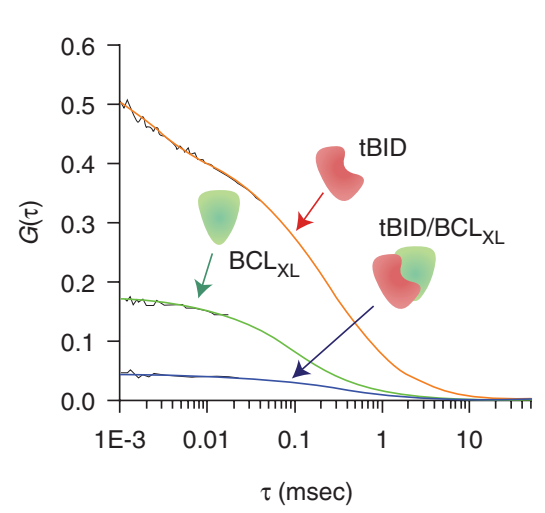

C

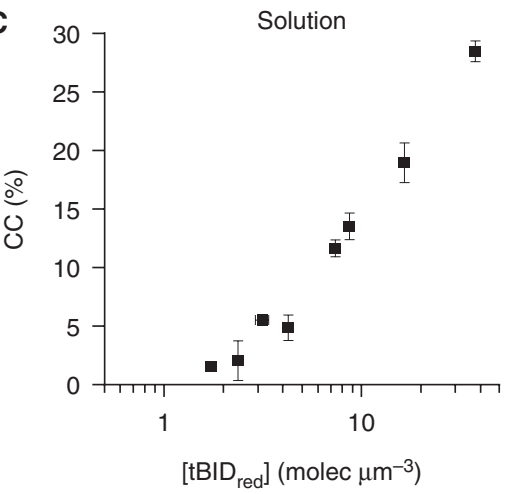

B

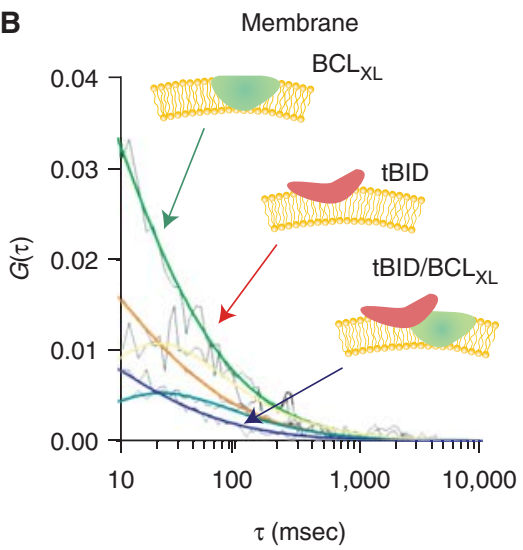

D

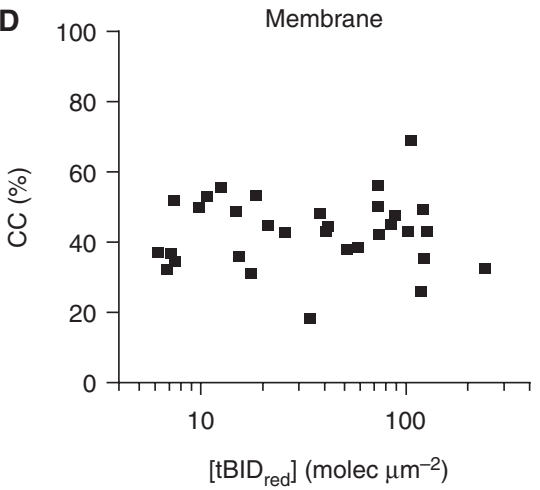

Figure 7. Stimulation of $\mathrm{tBID}$ and $\mathrm{BCL}_{\mathrm{XL}} \Delta \mathrm{Ct}$ intereaction by membrane. Cross-correlation in solution is affected by concentration $(A, C)$, whereas it is not changing and always higher in membrane $(B, D)$. (Image courtesy of Ana García-Sáez.)

undulations, or autofluorescent objects floating around. However, FCS derivatives like scanning FCS can overcome these problems to some extent, still preserving the high sensitivity and precision of the method. The main issue about FRAP seems to be the high laser power which may easily destroy the living sample and change the dynamics of the molecular system. Finally, FRAP gives information about immobile fractions whereas FCS cannot.

\section{Förster Resonance Energy Transfer (FRET)}

FRET is not really a technique, but rather a wellestablished photophysical phenomenon used to monitor molecular dynamics and interactions down to the single-molecule level. The idea behind FRET was first proposed by Theodor
Förster in 1948 (Förster 1948). The mechanism is based on the energy transfer of an excited fluorescent donor molecule to an acceptor molecule in a nonradiative fashion when they are in close proximity $(10-100 \AA)$.

The energy transfer not only depends on the distance between donor and acceptor, but also on the spectral properties of the dyes and the relative orientation of their transition dipole moments. The rate $\left(k_{\mathrm{t}}\right)$ and the efficiency $(E)$ of the energy transfer in FRET are given by

$$
\begin{aligned}
& k_{\mathrm{t}}=\frac{1}{\tau_{\mathrm{D}}}\left(R_{0} / r\right)^{6}, \\
& E=\frac{1}{1+\left(r / R_{0}\right)^{6}},
\end{aligned}
$$


where $\tau_{\mathrm{D}}$ is the donor lifetime in the absence of acceptor, $r$ is the spatial distance between donor and acceptor, and $R_{0}$ is the Förster distance of the donor/acceptor pair, which is the distance at which the energy transfer efficiency is $50 \%$. As seen, FRET efficiency highly depends on $R_{0}$, which is given by

$$
R_{0}=\left(\frac{9000 Q_{\mathrm{D}}(\ln 10) \kappa^{2} J(\lambda)}{128 \pi^{5} n^{4} N_{\mathrm{A}}}\right)^{1 / 6}
$$

where $Q_{D}$ is the quantum yield of the donor in the absence of the acceptor, $\kappa^{2}$ is the dipole orientation factor, $n$ is the refractive index of the medium, $N_{\mathrm{A}}$ is Avogadro's number, and $J$ is the spectral overlap between the emission spectrum of the donor and the absorption spectrum of the acceptor (Fig. 8). $J$ is calculated as

$$
J(\lambda)=\int f_{\mathrm{D}}(\lambda) \varepsilon_{\mathrm{A}}(\lambda) \lambda^{4} d \lambda,
$$

where $f_{\mathrm{D}}$ is the normalized donor emission spectrum, and $\varepsilon_{\mathrm{A}}$ is the acceptor molar extinction coefficient.

The dipole orientation factor $\kappa^{2}$ is often assumed to be $2 / 3$, which is valid when the acceptor and donor molecules are freely rotating, and considered to be isotropically oriented during the excited state lifetime. If the donor and acceptor molecules are not free to rotate, then this assumption is not valid anymore. In most cases, however, even modest reorientation of donor and acceptor molecules results in enough orientational averaging that $\kappa^{2}=2 / 3$ does not result in a large error in the estimated energy transfer distance, because of the sixthroot dependence of $R_{0}$ on $\kappa^{2}$. Even when $\kappa^{2}$ is quite different from $2 / 3$ the error can be associated with a shift in $R_{0}$, and thus, determinations of changes in relative distance for a particular system are still valid. Fluorescent proteins, for example, do not reorient on a timescale that is faster than their fluorescence lifetime. In this case, $0 \leq \kappa^{2} \leq 4$ is a valid approximation.

There are several ways to detect FRET. Acceptor emission can be detected on donor excitation (Gordon et al. 1998). Because the energy is transferred to the acceptor from the donor, the emission intensity of acceptor is expected to increase on donor excitation. Alternatively, the emission of the donor can be observed while photobleaching the acceptor molecule (Jovin and Arndtjovin 1989; Kenworthy and Edidin 1998; Wouters et al. 1998). When the acceptor is optically saturated, the excitation energy of the donor molecule is no longer used by the acceptor molecule, such that the emission intensity of donor molecule increases on acceptor photobleaching, directly proportional to the FRET rate.

Besides the fluorescence intensity measurements mentioned above, the fluorescence life time of the donor can be also measured. FLIMFRET is a technique to detect the decrease in the

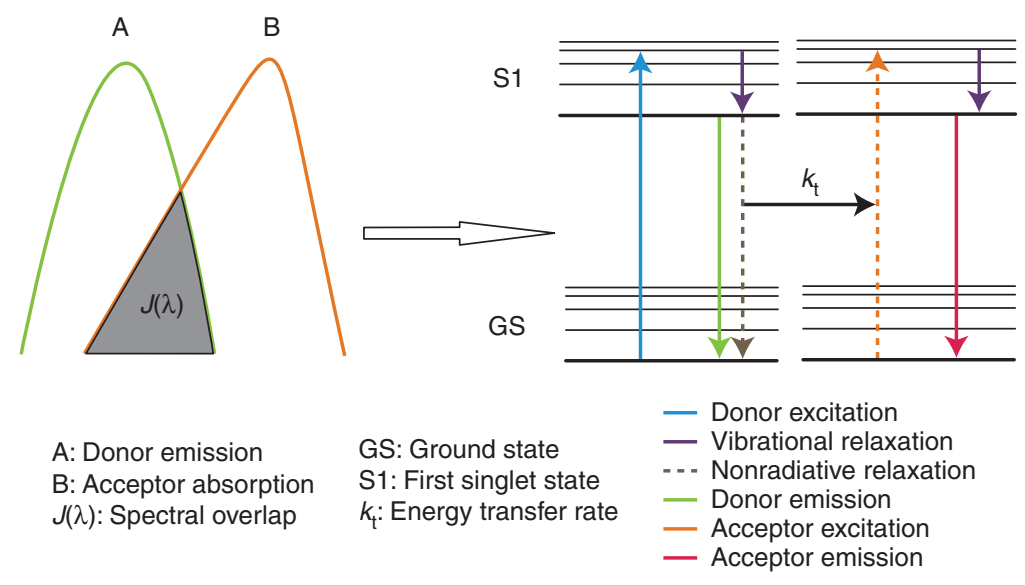

Figure 8. Principle of FRET. 
donor's fluorescence lifetime on energy transfer (Gadella and Jovin 1995; Bastiaens and Squire 1999). This method is quite insensitive to many artifacts which are going to be discussed later, but its main disadvantage is the more involved instrumental setup required to detect on nanosecond timescales. FLIM-FRETappears to be more vulnerable to some artifacts caused by $\mathrm{pH}$, temperature, and ionic strength of the medium, as these factors modify fluorescence lifetimes. However, with the proper controls, this method seems to be the most reliable one among others.

Fluorescence lifetime analysis can be performed in two ways, in time domain and in frequency domain. In the time domain approach, very short (picoseconds to femtoseconds) excitation pulses are used to excite the sample, and the lifetime is measured by collecting the resulting photons over time between the pulses, one by one. On the other hand, sinusoidally modulated light is used to excite the fluorophores in frequency domain. The emission is also sinusoidally modulated at the same frequency as the excitation, but there is a phase shift and reduction in the modulation depth, from which the fluorescence lifetime can be derived.

FRET can also be detected by fluorescence anisotropy (Runnels and Scarlata 1995; Gautier et al. 2001; Clayton et al. 2002; Lidke et al. 2003) which uses linearly polarized light to detect the orientation of the molecules. When there is no energy transfer, the orientation of excited molecule is highly correlated with the orientation of the emitting molecule. However, when there is FRET, the emitting molecules are not only the excited donors but also the acceptors, such that the correlation between the orientations of these two components will decrease remarkably. This method has a unique property to also determine FRET between identical molecules (so-called homo-FRET) which is very crucial in dimerization or oligomerization studies (Bader et al. 2009).

The FRET pair selection is an important issue. Theoretically, pairs are selected based on the spectral overlap criterion discussed above. The closer the spatial distance at closest proximity is expected to be, the larger the spectra may vary. Clearly, detection efficiency is maximized and cross talk minimized for spectrally more distinct probes. The most popular FRET pairs at present are GFP-RFP, CFP-YFP, BFP-GFP, GFP-mCherry for genetically modified proteins. There are also several classical FRET pairs based on chemical dyes, such as Cy3-Cy5 and Alexa488-Cy3. More FRET pairs and their properties can be found elsewhere (Sahoo et al. 2007).

\section{Difficulties and Delicacy}

In spite of its attractiveness of being a very intuitive technique with in principle rather straightforward experimental design and readout, FRET in praxis has many caveats to be considered carefully, some which are mentioned below.

To get reliable FRET results, spectral cross talk needs to be minimized. When the acceptor fluorescence intensity is used to assess FRET efficiency, spectral contamination always has to be corrected to some extent. Two major sources of this spectral contamination are the direct excitation of the acceptor, and the leakage of donor emission into the acceptor detection channel. As mentioned above, there is always a trade-off between minimal cross talk and a good spectral overlap factor $J$. The simplest way to correct for spectral contamination is to test the FRET signal in combinations of only acceptor, only donor and donor/acceptor combination, both with acceptor excitation and donor excitation.

Photoconversion is another problem often encountered with fluorescent proteins. Under certain conditions, emission spectra may change with time (e.g., on high laser power excitation) or sometimes spontaneously as a result of protein maturation. This is critical in FRET measurements, particularly when photobleaching is used as readout. It has been shown that on photobleaching of acceptor YFP, CFP-like emission is created without any FRET (Kirber et al. 2007).

Fluorescence lifetime-based FRET detection is insensitive to many of these problems as the life time is usually independent of excitation intensity. On the other hand, the big 
practical advantage of FRET as a straightforward and intuitive method to complement imaging is also lost, and the instrumentation gets much more involved.

The interactions of donor or acceptor molecules with other components in the medium should be carefully tested. If there is an unwanted binding of one or both to other molecules, this can produce positive and false negative results. Other problems may arise from too complicated stoichiometry (van den Bogaart et al. 2007) and the impossibility to attach fluorescent tags close enough to the interaction region (Miyawaki and Tsien 2000).

As for other fluorescence techniques, photobleaching should be avoided, as it usually changes the molecular ratio of donor and acceptor, resulting in artifacts of FRET efficiency. The donor should be particularly photostable long enough to transfer its energy. It should also exhibit low polarization anisotropy to eliminate the $\kappa^{2}$ deviations.

The brightness of donor and acceptor should ideally be comparable, otherwise resulting in saturation in one channel, or enhanced noise in the other channel due (Piston and Kremers 2007).

\section{Applications of FRET in Lipid Biology}

Being such a powerful tool to detect dynamic molecular interaction, FRET has had a strong impact on membrane and lipid research. Particularly, lipid/protein clusters in membranes are interesting topics of study, and there are many applications of FRET in this context. It has been shown that GPI-anchored proteins are enriched in cholesterol-dependent clusters, whereas some putative nonraft proteins are not. Cross-linking of GPI-anchored proteins affects the protein distribution on the membrane and their endocytosis, which highlights the role of immobile, actin-driven nanoclusters in the membrane (Varma and Mayor 1998; Sharma et al. 2004; Goswami et al. 2008). However, there are also some contradicting reports on similar systems, proposing that there are no functional clusters in the cell membrane (Kenworthy and Edidin 1999; Kenworthy et al.
2000; Glebov and Nichols 2004). FLIM-FRET was used to detect the effect of cholesterol depletion on lipid order (Grant et al. 2007) as well as dynamic protein-lipid interactions in live cells (Larijani et al. 2003). TIR-FRET was used to visualize protein-protein interactions on cell membranes and insulin secreting cells (Lam et al. 2010; Sohn et al. 2010). Two-photon FRET was applied to visualize protein-protein colocalization (Mills et al. 2003) and free versus clustered receptor-ligand complexes in the membrane (Wallrabe et al. 2003).

\section{Single-Particle Tracking (SPT)}

As discussed above, information on the diffusion of molecules in a membrane can usually be obtained by either FRAP or FCS. However, there are certain disadvantages to both techniques. The lateral resolution of both techniques is limited by the diffraction barrier. Additionally, both techniques have to average over many molecular events to obtain a reliable diffusion time. This averaging, however, masks potential heterogeneities in the diffusion characteristics, induced by, for example, molecular interactions or a heterogeneous membrane environment. Therefore, a technique providing access to the randomly distributed tracks of individual particles is greatly desirable. With the possibility of resolving single molecules, the spatial precision at which lateral detection can be performed is only determined by the number of photons it takes to compute a spotlike image, which can then be fitted with a point-spread function to determine its geometrical center (Toprak et al. 2007).

This approach, which has in past years gained tremendous impact because of the availability of extremely sensitive charge-coupled device (CCD) cameras, is known under the name of single-particle tracking (SPT). The underlying idea is that single particles or even molecules are followed by computer-enhanced video microscopy with a spatial resolution of tens of nanometers and a temporal resolution of tens of milliseconds. Thus, it is a suitable technique to investigate the diffusion characteristics of lipid or membrane-attached proteins, 
Fluorescence Techniques to Study Lipid Dynamics

as well as factors influencing lipid dynamics and membrane order.

The first SPT experiment was performed in 1982 by Barak and Webb to follow the lipoprotein receptor (Barak and Webb 1982). Then, nanovid microscopy was developed, in which molecules are labeled with gold nanoparticles which can be more easily tracked by wide-field microscopy (Debrabander et al. 1985, 1991). The technique was further improved in terms of resolution in later years (Schnapp et al. 1988; Sheetz and Kuo 1993). A large body of work on imaging and tracking of single lipid molecules on artificial and cell membranes was further catalyzed by the work by Schütz et al. (1997). Later on, the technique was further developed by to track the particles 3D with different strategies (Digman and Gratton 2009; Katayama et al. 2009; Ragan et al. 2006).

The idea behind the technique is to follow the single-molecule movement over time, record it as trajectories and analyze these trajectories according to the diffusion theory. The main way to analyze the trajectories is to calculate the mean square displacement (MSD) defined as

$$
\begin{aligned}
\operatorname{MSD}(\tau)= & \left\langle(x(t)-x(t+\tau))^{2}+(y(t)-y(t+\tau))^{2}\right. \\
& \left.+(z(t)-z(t+\tau))^{2}\right\rangle,
\end{aligned}
$$

where $x, y$, and $z$ are the coordinates of the particles, $\tau$ is the lag time, and \langle\rangle represents the temporal averaging.

The MSD represents the average distance that the molecule travels during the lag time and is thus directly related to the local mobility of the molecule. Once the trajectories are recorded and the MSD is obtained experimentally, it can be compared with theoretical models as listed in Table 3.

Of particular importance in membranes are usually the cases of subdiffusion or confined diffusion, which can be induced by corralling of the diffusing molecules in domains, or any other interactions slowing them down locally. To resolve the dynamics of small local constraints, one trajectory can be divided into different parts, to display the changes in mobility over time.
Table 3. SPT fitting models

\begin{tabular}{ll}
\hline Type of diffusion & \multicolumn{1}{c}{ Model } \\
\hline 3D free diffusion & $6 D t$ \\
3D anomalous subdiffusion & $6 D t^{\alpha}$ \\
3D diffusion with directed & $6 D t+(V t)^{2}$ \\
$\quad$ motion & $4 D t$ \\
2D free diffusion & $4 D t^{\alpha}$ \\
2D anomalous subdiffusion & $4 D t+(V t)^{2}$ \\
2D diffusion with directed & \\
$\quad$ motion & $r\left(1-A_{1} e^{\left(4 A_{2} D t / r\right)}\right)$ \\
2D corralled motion
\end{tabular}

Instrumentally, SPT can be performed by regular wide-field microscopy, as well as confocal, two-photon, and TIRF. The selection of the illumination method depends on the application.

Different labels can be used in SPT, according to the nature of the experiment and the temporal and spatial scales to be observed. Usually, the spatial precision scales with the signal-to-noise ratio that can be reached, but bright probes such as nanoparticles and beads have to be used with care, as they might also influence the mobility of molecules. The most commonly used labels are gold nanoparticles, quantum dots, and fluorescent microspheres. The most convenient way of labeling is to conjugate the probes with antibodies or adaptor proteins which specifically target the molecule of interest.

After the particle location is accurately determined in $x-y$ or $x-y-z$ (Levi and Gratton 2007), different algorithms can be applied to acquire the full trajectories. The basic algorithms are cross-correlating subsequent images (Gelles et al. 1988; Kusumi et al. 1993), calculating the center-of-mass of the labeled object (Lee et al. 1991; Ghosh and Webb 1994), or directly fitting the image to a Gaussian distribution (Anderson et al. 1992; Schutz et al. 1997). The correlation method compares the image with the Kernel of the successive image. This method gives the best performance at low signal-to-noise ratios. The centroid (center-ofmass) algorithm compares the center of mass of two subsequent images to determine the distance the molecule has travelled in between. A Gaussian fit algorithm directly fits the image of the object to a 2D Gaussian distribution 
(as an approximation of the PSF):

$$
G(x, y)=A \exp \left[-\frac{\left(x-x_{0}\right)^{2}+\left(y-y_{0}\right)^{2}}{B}\right] \text {. }
$$

This algorithm shows the greatest performance when the object is of subwavelength size. A detailed comparison of these algorithms, their potential problems, and performances can be found in Cheezum et al. (2001). There are also algorithms available which combine the correlation and Gaussian fit algorithms (Levi et al. 2006a,b).

\section{Difficulties and Delicacy}

There are many aspects to be taken into account to perform meaningful SPT analysis.

First, when using fluorescent probes rather than nanoparticles or beads, photodamage should be minimized. SPT requires a long time to record the trajectories. Therefore, photobleaching can be an important issue. The photostability of quantum dots or fluorescent beads is usually quite high, but when fluorescent lipid analogs or fluorescent proteins are used, the intensity has to be much reduced, limiting the spatial resolution. A very important strategy is to illuminate only while recording the photons, and shutting the laser during camera readout (i.e., frame transfer to the data storage).

A big argument in the SPT field is the effect of large labels on molecular motion. When a bead or nanoparticle is attached to small molecules such as lipids, the diffusion characteristics may change dramatically. Moreover, linkers to attach the label to the molecule can also be problematic. Generally, short linkers and small labels are desirable. Detailed work on this issue can be found in the literature (Dahan et al. 2003).

The determination of particle location is the most crucial aspect in SPT, and much work has been devoted to maximize the spatial precision. The accuracy of the spatial coordinates was in the last years improved drastically, down to $1.5 \mathrm{~nm}$ in recent studies (Yildiz et al. 2003, 2004a,b).

The tracking software must be sensitive to the particle brightness changes, because of changes in the particle location with respect to the focal plane. This effect can also be used to record $z$ coordinates with moderate precision (Levi et al. 2005; Ragan et al. 2006). Needless to say, cameras should be fast and sensitive enough to catch the single-molecule signal.

The trajectory algorithm should be carefully chosen, taking all the advantages and disadvantages into consideration. The Gaussian fit algorithm, for instance, seems easy and robust to apply, but it just uses the brightest point as the center and does not take the topographical structure into account. Therefore, it may result in wrong results depending on the molecule topology (Cheezum et al. 2001).

The lag time $\tau$ used to record the MSD is also an important variable. It is supposed to be lower than one-fourth of the total trajectory time (Saxton and Jacobson 1997).

Scattering or autofluorescence background is usually quite low, but it decreases the localization accuracy of SPT. It is more dominant when the centroid algorithm is used, because centerof-mass is biased notably by the background (Cheezum et al. 2001; Levi and Gratton 2007).

\section{Applications}

SPT hfas been applied successfully to investigate different diffusion mechanisms and establish the models for active transport, free diffusion, anomalous diffusion, and confined diffusion, as seen in Figure 9 and Table 3 (Saxton 1994a,b,

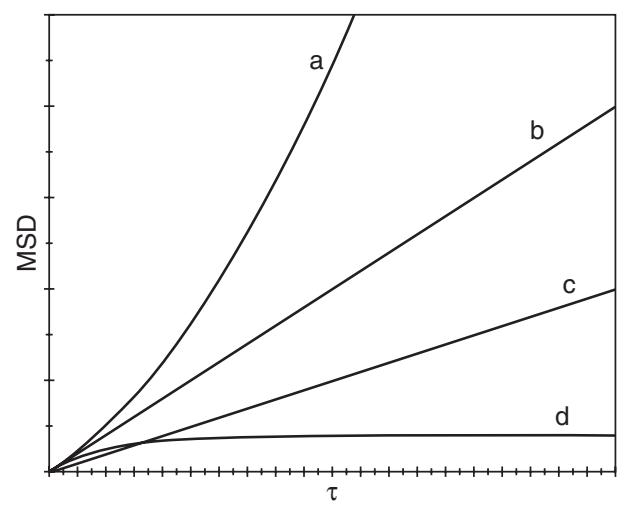

Figure 9. SPT models. (a) Active transport, (b) free diffusion, (c) anomalous diffusion, and (d) confined diffusion. 
1995, 1996a,b, 1997; Saxton and Jacobson 1997). Lipid diffusion is found to be highly restricted in native plasma membranes, unlike in liposomes, highlighting the role of cytoskeleton in membrane dynamics (Kusumi et al. 2005). Another diffusion type was described according to these observations, called hop diffusion. It is claimed that both lipids and trans-membrane proteins diffuse in small compartments whose boundaries were mostly determined by cytoskeleton of the cell until they change the compartment by hop diffusion (Tomishige et al. 1998). Although there are many studies claiming that saturated lipids and GPI-anchored proteins show confined diffusion on cell membrane on islands of $80 \mathrm{~nm}$ and $700 \mathrm{~nm}$ in size (Schutz et al. 2000; Lenne et al. 2006; Wenger et al. 2007), some other SPT studies has recently claimed that the diffusion of saturated and unsaturated lipids is not different in cell membrane, which can be because of very small size $(<16 \mathrm{~nm})$ or very fast association/dissociation of the rafts. Recently, two-color SPT (Dunne et al. 2009) and micropatterning (Schwarzenbacher et al. 2008) was established to visualize the colocalization on a single-molecule level. A concise summary on many applications can be found elsewhere (Saxton and Jacaobson 1997; Levi and Gratton 2007).

\section{ACKNOWLEDGMENTS}

We would like to thank Senthil Arumugan, Fabian Heinemann, Grzegorz Chwastek, and Harekrushna Sahoo for their careful reading of and valuable comments on the manuscript.

\section{REFERENCES}

Amos WB, White JG. 2003. How the confocal laser scanning microscope entered biological research. Biol Cell 95: 335-342.

Anderson CM, Georgiou GN, Morrison IEG, Stevenson GVW, Cherry RJ. 1992. Tracking of cell-surface receptors by fluorescence digital imaging microscopy using a charge-coupled device camera-Low-density-lipoprotein and influenza-virus receptor mobility at $4{ }^{\circ} \mathrm{C}$. J Cell Sci 101: 415-425.

Aoki R, Kitaguchi T, Oya M, Yanagihara Y, Sato M, Miyawaki A, Tsuboi T. 2010. Duration of fusion pore opening and the amount of hormone released are regulated by myosin II during kiss-and-run exocytosis. Biochem J 429: 497504.

Axelrod D. 1977. Cell-surface heating during fluorescence photobleaching recovery experiments. Biophys $J \mathbf{1 8}$ 129-131.

Axelrod D. 2008. Chapter 7: Total internal reflection fluorescence microscopy. Methods Cell Biology 89: 169-221.

Axelrod D, Thompson NL, Burghardt TP. 1983. Total internal reflection fluorescent microscopy. J Microsc 129: $19-28$.

Axelrod D, Koppel DE, Schlessinger J, Elson E, Webb WW. 1976. Mobility measurement by analysis of fluorescence photobleaching recovery kinetics. Biophys J 16: 10551069.

Bacia K, Schwille P, Kurzchalia T. 2005. Sterol structure determines the separation of phases and the curvature of the liquid-ordered phase in model membranes. Proc Natl Acad Sci 102: 3272-3277.

Bacia K, Scherfeld D, Kahya N, Schwille P. 2004. Fluorescence correlation spectroscopy relates rafts in model and native membranes. Biophys J 87: 1034-1043.

Bader AN, Hofman EG, Voortman J, Henegouwen P, Gerritsen HC. 2009. Homo-FRET imaging enables quantification of protein cluster sizes with subcellular resolution. Biophys J 97: 2613-2622.

Bagatolli LA. 2003. Direct observation of lipid domains in free standing bilayers: From simple to complex lipid mixtures. Chem Phys Lipids 122: 137-145.

Bagatolli LA, Gratton E. 1999. Two-photon fluorescence microscopy observation of shape changes at the phase transition in phospholipid giant unilamellar vesicles. Biophys J 77: 2090-2101.

Bagatolli LA, Gratton E. 2000a. Two photon fluorescence microscopy of coexisting lipid domains in giant unilamellar vesicles of binary phospholipid mixtures. Biophys J 78: 290-305.

Bagatolli LA, Gratton E. 2000b. A correlation between lipid domain shape and binary phospholipid mixture composition in free standing bilayers: A two-photon fluorescence microscopy study. Biophys J 79: 434-447.

Bagatolli LA, Sanchez SA, Hazlett T, Gratton E. 2003. Giant vesicles, laurdan, and two-photon fluorescence microscopy: Evidence of lipid lateral separation in bilayers. Biophotonics 360: 481-500.

Barak LS, Webb WW. 1982. Diffusion of low density lipoprotein-receptor complex on human fibroblasts. J Cell Biol 95: 846-852.

Bastiaens PIH, Squire A. 1999. Fluorescence lifetime imaging microscopy: Spatial resolution of biochemical processes in the cell. Trends Cell Biol 9: 48-52.

Baumgart T, Hunt G, Farkas ER, Webb WW, Feigenson GW. 2007. Fluorescence probe partitioning between $\mathrm{L}_{\mathrm{o}} / \mathrm{L}_{\mathrm{d}}$ phases in lipid membranes. Biochim Biophys Acta 1768: 2182-2194.

Benda A, Benes M, Marecek V, Lhotsky A, Hermens WT, Hof M. 2003. How to determine diffusion coefficients in planar phospholipid systems by confocal fluorescence correlation spectroscopy. Langmuir 19: 4120-4126. 
Bockmann RA, Hac A, Heimburg T, Grubmuller H. 2003. Effect of sodium chloride on a lipid bilayer. Biophys J 85: $1647-1655$.

Boldyrev IA, Zhai XH, Momsen MM, Brockman HL, Brown RE, Molotkovsky JG. 2007. New BODIPY lipid probes for fluorescence studies of membranes. J Lipid Res 48: $1518-1532$.

Brakenhoff GJ, Blom P, Barends P. 1979. Confocal scanning light-microscopy with high aperture immersion lenses. J Microsc 117: 219-232.

Byrne GD, Pitter MC, Zhang J, Falcone FH, Stolnik S, Somekh MG. 2008. Total internal reflection microscopy for live imaging of cellular uptake of sub-micron nonfluorescent particles. J Microsc 231: 168-179.

Cannon B, Weaver N, Pu QS, Thiagarajan V, Liu SR, Huang JY, Vaughn MW, Cheng KH. 2005. Cholesterol modulated antibody binding in supported lipid membranes as determined by total internal reflectance microscopy on a microfabricated high-throughput glass chip. Langmuir 21: 9666-9674.

Cheezum MK, Walker WF, Guilford WH. 2001. Quantitative comparison of algorithms for tracking single fluorescent particles. Biophys J 81: 2378-2388.

Chiantia S, Kahya N, Schwille P. 2007. Raft domain reorganization driven by short- and long-chain ceramide: A combined AFM and FCS study. Langmuir 23: 7659-7665.

Chiantia S, Ries J, Schwille P. 2009. Fluorescence correlation spectroscopy in membrane structure elucidation. Biochim Biophys Acta 1788: 225-233.

Chiantia S, Ries J, Chwastek G, Carrer D, Li Z, Bittman R, Schwille P. 2008. Role of ceramide in membrane protein organization investigated by combined AFM and FCS. Biochim Biophys Acta 1778: 1356-1364.

Choucair A, Chakrapani M, Chakravarthy B, Katsaras J, Johnston LJ. 2007. Preferential accumulation of A $\beta(1-42)$ on gel phase domains of lipid bilayers: An AFM and fluorescence study. Biochim Biophys Acta 1768: $146-154$.

Clayton AHA, Hanley QS, Arndt-Jovin DJ, Subramaniam V, Jovin TM. 2002. Dynamic fluorescence anisotropy imaging microscopy in the frequency domain (rFLIM). Biophys J 83: 1631-1649.

Coban O, Burger M, Laliberte M, Ianoul A, Johnston LJ. 2007. Ganglioside partitioning and aggregation in phaseseparated monolayers characterized by bodipy GM1 monomer/dimer emission. Langmuir 23: 6704-6711.

Dahan M, Levi S, Luccardini C, Rostaing P, Riveau B, Triller A. 2003. Diffusion dynamics of glycine receptors revealed by single-quantum dot tracking. Science 302: 442-445.

Davidovi P, Egger MD. 1973. Photomicrography of corneal endothelial cells in-vivo. Nature 244: 366-367.

Davis LM, Shen. 2006. Accounting for triplet and saturation effects in FCS measurements. Curr Pharm Biotechnol 7: 287-301.

Debrabander M, Geuens G, Nuydens R, Moeremans M, Demey J. 1985. Probing microtubule-dependent intracellular motility with nanometer particle video ultramicroscopy (nanovid ultramicroscopy). Cytobios 43: 273-283.

Debrabander M, Nuydens R, Ishihara A, Holifield B, Jacobson K, Geerts H. 1991. Lateral diffusion and retrograde movements of individual cell-surface components on single motile cells observed with nanovid microscopy. J Cell Biol 112: 111-124.

Delon A, Usson Y, Derouard J, Biben T, Souchier C. 2004. Photobleaching, mobility, and compartmentalisation: Inferences in fluorescence correlation spectroscopy. J Fluorescence 14: 255-267.

Denk W, Strickler JH, Webb WW. 1990. 2-photon laser scanning fluorescence microscopy. Science 248: 73-76.

Dertinger T, Pacheco V, von der Hocht I, Hartmann R, Gregor I, Enderlein J. 2007. Two-focus fluorescence correlation spectroscopy: A new tool for accurate and absolute diffusion measurements. Chemphyschem 8: 433-443.

Dewa T, Sugiura R, Suemori Y, Sugimoto M, Takeuchi T, Hiro A, Iida K, Gardiner AT, Cogdell RJ, Nango M. 2006. Lateral organization of a membrane protein in a supported binary lipid domain: Direct observation of the organization of bacterial light-harvesting complex 2 by total internal reflection fluorescence microscopy. Langmuir 22: 5412-5418.

Dietrich C, Bagatolli LA, Volovyk ZN, Thompson NL, Levi M, Jacobson K, Gratton E. 2001. Lipid rafts reconstituted in model membranes. Biophys J 80: 1417-1428.

Digman MA, Gratton E. 2009. Imaging barriers to diffusion by pair correlation functions. Biophys J 97: 665-673.

Dittrich PS, Schwille P. 2001. Photobleaching and stabilization of fluorophores used for single-molecule analysis with one- and two-photon excitation. Appl Phys B 73: 829-837.

Doeven MK, Folgering JHA, Krasnikov V, Geertsma ER, van den Bogaart G, Poolman B. 2005. Distribution, lateral mobility and function of membrane proteins incorporated into giant unilamellar vesicles. Biophys $J$ 88: $1134-1142$.

Dunne PD, Fernandes RA, McColl J, Yoon JW, James JR, Davis SJ, Klenerman D. 2009. DySCo: Quantitating associations of membrane proteins using two-color singlemolecule tracking. Biophys J 97: L5-L7.

Edidin M. 1992. Patches, posts and fences: Proteins and plasma membrane domains. Trends Cell Biol 2: 376-380.

Egger MD, Petran M. 1967. New reflected-light microscope for viewing unstained brain and ganglion cells. Science 157: 305-307.

Eggeling C, Ringemann C, Medda R, Schwarzmann G, Sandhoff K, Polyakova S, Belov VN, Hein B, von Middendorff C, Schonle A, et al. 2009. Direct observation of the nanoscale dynamics of membrane lipids in a living cell. Nature 457: U1159-U1121.

Eigen M, Rigler R. 1994. Sorting single molecules-Application to diagnostics and evolutionary biotechnology. Proc Natl Acad Sci 91: 5740-5747.

Ellenberg J, Siggia ED, Moreira JE, Smith CL, Presley JF, Worman HJ, Lippincott-Schwartz J. 1997. Nuclear membrane dynamics and reassembly in living cells: Targeting of an inner nuclear membrane protein in interphase and mitosis. J Cell Biol 138: 1193-1206.

Elson EL, Magde D. 1974. Fluorescence correlation spectroscopy. 1. Conceptual basis and theory. Biopolymers 13: $1-27$. 
Enderlein J, Gregor I, Patra D, Dertinger T, Kaupp UB. 2005 Performance of fluorescence correlation spectroscopy for measuring diffusion and concentration. Chemphyschem 6: $2324-2336$.

Enderlein J, Gregor I, Patra D, Fitter J. 2004. Art and artefacts of fluorescence correlation spectroscopy. Curr Pharmaceut Biotech 5: 155-161.

Engelke M, Bojarski P, Bloss R, Diehl H. 2001. Tamoxifen perturbs lipid bilayer order and permeability: Comparison of DSC, fluorescence anisotropy, Laurdan generalized polarization and carboxyfluorescein leakage studies. Biophys Chem 90: 157-173.

Feder TJ, BrustMascher I, Slattery JP, Baird B, Webb WW. 1996. Constrained diffusion or immobile fraction on cell surfaces: A new interpretation. Biophysical $J$ 70: 2767-2773.

Förster T. 1948. Intermolecular energy migration and fluorescence. Ann Phys 2: 55-75.

Fox CB, Wayment JR, Myers GA, Endicott SK, Harris JM. 2009. Single-molecule fluorescence imaging of peptide binding to supported lipid bilayers. Anal Chem 81: 5130-5138.

Fulbright RM, Axelrod D. 1993. Dynamics of nonspecific adsorption of insulin to erythrocyte membrane. J Fluor 3: $1-16$.

Gadella TWJ, Jovin TM. 1995. Oligomerization of epidermal growth-factor receptors on a431 cells studied by time-resolved fluorescence imaging microscopy-A stereochemical model for tyrosine kinase receptor activation. J Cell Biol 129: 1543-1558.

García-Sáez AJ, Schwille P. 2010. Stability of lipid domains. FEBS Lett 584: 1653-1658.

García-Sáez AJ, Ries J, Orzaez M, Perez-Paya E, Schwille P. 2009. Membrane promotes tBID interaction with BCLXL. Nat Struct Mol Biol 16: U1178-U1179.

García-Sáez AJ, Carrer DC, Schwille P. 2010. Fluorescence correlation spectroscopy for the study of membrane dynamics and organization in giant unilamellar vesicles. Meth Mol Biol 606: 493-508.

Gasecka A, Han TJ, Favard C, Cho BR, Brasselet S. 2009. Quantitative imaging of molecular order in lipid membranes using two-photon fluorescence polarimetry. Biophys J 97: 2854-2862.

Gautier I, Tramier M, Durieux C, Coppey J, Pansu RB, Nicolas JC, Kemnitz K, Coppey-Moisan M. 2001. HomoFRET microscopy in living cells to measure monomerdimer transition of GFP-tagged proteins. Biophys $J$ 80: 3000-3008.

Gelles J, Schnapp BJ, Sheetz MP. 1988. Tracking kinesindriven movements with nanometre-scale precision. Nature 331: 450-453.

Ghosh RN, Webb WW. 1994. Automated detection and tracking of individual and clustered cell-surface lowdensity-lipoprotein receptor molecules. Biophys $J$ 66: 1301-1318.

Gidwani A, Holowka D, Baird B. 2001. Fluorescence anisotropy measurements of lipid order in plasma membranes and lipid rafts from RBL-2H3 mast cells. Biochemistry 40: $12422-12429$.
Fluorescence Techniques to Study Lipid Dynamics

Glebov OO, Nichols BJ. 2004. Lipid raft proteins have a random distribution during localized activation of the T-cell receptor. Nat Cell Biol 6: 238-243.

Goodwin JS, Drake KR, Remmert CL, Kenworthy AJ. 2005. Ras diffusion is sensitive to plasma membrane viscosity. Biophys J 89: 1398-1410.

Goppert M. 1929. Über die Wahrscheinlichkeit des Zusammenwirkens zweier Lichtquanten in einem Elementarakt. Naturwissenschaften 17: 932-932.

Gordon GW, Berry G, Liang XH, Levine B, Herman B. 1998. Quantitative fluorescence resonance energy transfer measurements using fluorescence microscopy. Biophys $J$ 74: $2702-2713$.

Gordon GW, Chazotte B, Wang XF, Herman B. 1995. Analysis of simulated and experimental fluorescence recovery after photobleaching. Data for two diffusing components. Biophys J 68: 766-778.

Gorg B, Morwinsky A, Keitel V, Qvartskhava N, Schror K, Haussinger D. 2010. Ammonia triggers exocytotic release of L-glutamate from cultured rat astrocytes. Glia 58: 691-705.

Goswami D, Gowrishankar K, Bilgrami S, Ghosh S, Raghupathy R, Chadda R, Vishwakarma R, Rao M, Mayor S. 2008. Nanoclusters of GPI-anchored proteins are formed by cortical actin-driven activity. Cell 135: 1085-1097.

Grant DM, McGinty J, McGhee EJ, Bunney TD, Owen DM, Talbot CB, Zhang W, Kumar S, Munro I, Lanigan PMP, et al. 2007. High speed optically sectioned fluorescence lifetime imaging permits study of live cell signaling events. Opt Exp 15: 15656-15673.

Gregor I, Patra D, Enderlein J. 2005. Optical saturation in fluorescence correlation spectroscopy under continuouswave and pulsed excitation. Chemphyschem 6: 164-170.

Guo L, Har JY, Sankaran J, Hong YM, Kannan B, Wohland T. 2008. Molecular diffusion measurement in lipid bilayers over wide concentration ranges: A comparative study. Chemphyschem 9: 721-728.

Hamilton DK, Wilson T. 1986. Scanning optical microscopy by objective lens scanning. J Phys E-Scientific Instruments 19: $52-54$.

Hao MM, Mukherjee S, Maxfield FR. 2001. Cholesterol depletion induces large scale domain segregation in living cell membranes. Proc Natl Acad Sci 98: 13072-13077.

Haupts U, Maiti S, Schwille P, Webb WW. 1998. Dynamics of fluorescence fluctuations in green fluorescent protein observed by fluorescence correlation spectroscopy. Proc Natl Acad Sci 95: 13573-13578.

Hein B, Willig KI, Hell SW. 2008. Stimulated emission depletion (STED) nanoscopy of a fluorescent proteinlabeled organelle inside a living cell. Proc Natl Acad Sci 105: $14271-14276$.

Heinze KG, Koltermann A, Schwille P. 2000. Simultaneous two-photon excitation of distinct labels for dual-color fluorescence crosscorrelation analysis. Proc Natl Acad Sci 97: 10377-10382.

Heinze KG, Rarbach M, Jahnz M, Schwille P. 2002. Twophoton fluorescence coincidence analysis: Rapid measurements of enzyme kinetics. Biophys J 83: 1671-1681.

Hellen E, Axelrod D. 1991. Kinetics of epidermal growth factor/receptor binding on cells measured by total internal 
reflection/fluorescence recovery after photobleaching J Fluor 1: 113-128.

Hellwarth R, Christensen P. 1975. Nonlinear optical microscope using second-harmonic generation. Appl Opt 14: 247-248.

Hess ST, Webb WW. 2002. Focal volume optics and experimental artifacts in confocal fluorescence correlation spectroscopy. Biophys J 83: 2300-2317.

Holtta-Vuori M, Uronen FL, Repakova J, Salonen E, Vattulainen I, Panula P, Li ZG, Bittman R, Ikonen E. 2008. BODIPY-cholesterol: A new tool to visualize sterol trafficking in living cells and organisms. Traffic 9: 1839-1849.

Honerkamp-Smith AR, Cicuta P, Collins MD, Veatch SL, den Nijs M, Schick M, Keller SL. 2008. Line tensions, correlation lengths, and critical exponents in lipid membranes near critical points. Biophys J 95: 236-246.

Honigmann A, Walter C, Erdmann F, Eggeling C, Wagner R. 2010. Characterization of horizontal lipid bilayers as a model system to study lipid phase separation. Biophys $J$ 98: 2886-2894.

Humpolickova J, Benda A, Enderlein J. 2009. Optical saturation as a versatile tool to enhance resolution in confocal microscopy. Biophys J 97: 2623-2629.

Humpolickova J, Gielen E, Benda A, Fagulova V, Vercammen J, Vandeven M, Hof M, Ameloot M, Engelborghs Y. 2006. Probing diffusion laws within cellular membranes by Z-scan fluorescence correlation spectroscopy. Biophys J 91: L23-L25.

Icenogle RD, Elson EL. 1983a. Fluorescence correlation spectroscopy and photobleaching recovery of multiple binding reactions. 1. Theory and FCS measurements. Biopolymers 22: 1919-1948.

Icenogle RD, Elson EL. 1983b. Fluorescence correlation spectroscopy and photobleaching recovery of multiple binding reactions. 2. FPR AND FCS measurements at low and high DNA concentrations. Biopolymers 22: 1949-1966.

Ira S Zou, Ramirez DMC, Vanderlip S, Ogilvie W, Jakubek ZJ, Johnston LJ. 2009. Enzymatic generation of ceramide induces membrane restructuring: Correlated AFM and fluorescence imaging of supported bilayers. J Struct Biol 168: 78-89.

Jacobson K, Sheets ED, Simson R. 1995. Revisiting the fluid mosaic model of membranes. Science 268: 1441-1442.

Jin L, Millard AC, Wuskell JP, Dong XM, Wu DQ, Clark HA, Loew LM. 2006. Characterization and application of a new optical probe for membrane lipid domains. Biophys J 90: $2563-2575$.

Johnson SA, Stinson BM, Go MS, Carmona LM, Reminick JI, Fang X, Baumgart T. 2010. Temperature-dependent phase behavior and protein partioning in giant plasma membrane vesicles. Biochim Biophys Acta 1798: 1427-1435.

Jorgensen L, Wood GK, Rosenkrands I, Petersen C, Christensen D. 2009. Protein adsorption and displacement at lipid layers determined by total internal reflection fluorescence (TIRF). J Lipo Res 19: 99-104.

Joselevitch C, Zenisek D. 2009. Imaging exocytosis in retinal bipolar cells with TIRF microscopy. J Vis Exp 9: 1305.
Jovin TM, Arndtjovin DJ. 1989. Luminescence digital imaging microscopy. Annu Rev Biophys Biophys Chem 18: 271-308.

Juhasz J, Davis JH, Sharom FJ. 2010. Fluorescent probe partitioning in giant unilamellar vesicles of 'lipid raft' mixtures. Biochem J 430: 415-423.

Kaiser W, Garrett CGB. 1961. Two-photon excitation in $\mathrm{CaF}_{2}: \mathrm{Eu}^{2+}$. Phys Rev Lett 7: 229.

Kahya N, Schwille P. 2006a. Fluorescence correlation studies of lipid domains in model membranes (Review). Mol Membr Biol 23: 29-39.

Kahya N, Schwille P. 2006b. How phospholipid-cholesterol interactions modulate lipid lateral diffusion, as revealed by fluorescence correlation spectroscopy. J Fluorescence 16: $671-678$.

Kahya N, Scherfeld D, Bacia K, Poolman B, Schwille P. 2003. Probing lipid mobility of raft-exhibiting model membranes by fluorescence correlation spectroscopy. J Biol Chem 278: 28109-28115.

Kahya N, Scherfeld D, Bacia K, Schwille P. 2004. Lipid domain formation and dynamics in giant unilamellar vesicles explored by fluorescence correlation spectroscopy. J Struct Biol 147: 77-89.

Kaiser HJ, Lingwood D, Levental I, Sampaio JL, Kalvodova L, Rajendran L, Simons K. 2009. Order of lipid phases in model and plasma membranes. Proc Natl Acad Sci 106: $16645-16650$.

Katayama Y, Burkacky O, Meyer M, Brauchle C, Gratton E, Lamb DC. 2009. Real-time nanomicroscopy via threedimensional single-particle tracking. Chemphyschem 10: $2458-2464$.

Kenworthy AK. 2005. Fleeting glimpses of lipid rafts: How biophysics is being used to track them. J Invest Med 53: 312-317.

Kenworthy AK. 2007. Fluorescence recovery after photobleaching studies of lipid rafts. Methods Mol Biol 398: 179-192.

Kenworthy AK, Edidin M. 1998, Distribution of a glycosylphosphatidylinositol-anchored protein at the apical surface of MDCK cells examined at a resolution of $<100$ angstrom using imaging fluorescence resonance energy transfer. J Cell Biol 142: 69-84.

Kenworthy AK, Edidin M. 1999. Imaging fluorescence resonance energy transfer as probe of membrane organization and molecular associations of GPI-anchored proteins. Methods Mol Biol 116: 37-49.

Kenworthy AK, Nichols BJ, Remmert CL, Hendrix GM, Kumar M, Zimmerberg J, Lippincott-Schwartz J. 2004. Dynamics of putative raft-associated proteins at the cell surface. J Cell Biol 165: 735-746.

Kenworthy AK, Petranova N, Edidin M. 2000. Highresolution FRET microscopy of cholera toxin B-subunit and GPI-anchored proteins in cell plasma membranes. Mol Biol Cell 11: 1645-1655.

Kim SA, Heinze KG, Schwille P. 2007. Fluorescence correlation spectroscopy in living cells. Nat Methods 4: 963-973.

Kim HM, Choo HJ, Jung SY, Ko YG, Park WH, Jeon SJ, Kim CH, Joo TH, Cho BR. 2007. A two-photon fluorescent probe for lipid raft imaging: C-laurdan. Chembiochem 8: 553-559. 
Kim HM, Jeong BH, Hyon JY, An MJ, Seo MS, Hong JH, Lee KJ, Kim CH, Joo TH, Hong SC, et al. 2008. Two-photon fluorescent turn-on probe for lipid rafts in live cell and tissue. J Am Chem Soc 130: 4246-4247.

Kirber MT, Chen K, Keaney JF. 2007. YFP photoconversion revisited: Confirmation of the CFP-like species. Nat Methods 4: 767-768.

Koppel DE, Axelrod D, Schlessinger J, Elson EL, Webb WW. 1976. Dynamics of fluorescence marker concentration as a probe of mobility. Biophys J 16: 1315-1329.

Korlach J, Schwille P, Webb WW, Feigenson GW. 1999. Characterization of lipid bilayer phases by confocal microscopy and fluorescence correlation spectroscopy. Proc Natl Acad Sci 96: 8461-8466.

Kuerschner L, Ejsing CS, Ekroos K, Shevchenko A, Anderson KI, Thiele C.2005. Polyene-lipids: A new tool to image lipids. Nat Methods 2: 39-45.

Klymchenko AS, Oncul S, Didier P, Schaub E, Bagatolli L, Duportail G, Mely Y. 2009. Visualization of lipid domains in giant unilamellar vesicles using an environment-sensitive membrane probe based on 3-hydroxyflavone. Biochim Biophys Acta 1788: 495-499.

Kusumi A, Sako Y, Yamamoto M. 1993. Confined lateral diffusion of membrane-receptors as studied by singleparticle tracking (NANOVID microscopy)_Effects of calcium-induced differentiation in cultured epithelialcells. Biophys J 65: 2021-2040.

Kusumi A, Nakada C, Ritchie K, Murase K, Suzuki K, Murakoshi H, Kasai RS, Kondo J, Fujiwara T. 2005. Paradigm shift of the plasma membrane concept from the twodimensional continuum fluid to the partitioned fluid: High-speed single-molecule tracking of membrane molecules. Annu Rev Biophys Biomol Struct 34: U351-U354.

Lagerholm BC, Weinreb GE, Jacobson K, Thompson NL. 2005. Detecting microdomains in intact cell membranes. Annu Rev Phys Chem 56: 309-336.

Lam AD, Ismail S, Wu R, Yizhar O, Passmore DR, Ernst SA, Stuenkel EL. 2010. Mapping dynamic protein interactions to insulin secretory granule behavior with TIRFFRET. Biophys J 99: 1311-1320.

Larijani B, Allen-Baume V, Morgan CP, Li M, Cockcroft S. 2003. EGF regulation of PITP dynamics is blocked by inhibitors of phospholipase $\mathrm{C}$ and of the Ras-MAP kinase pathway. Curr Biol 13: 78-84.

Lee GM, Ishihara A, Jacobson KA. 1991. Direct observation of Brownian-motion of lipids in a membrane. Proc Natl Acad Sci 88: 6274-6278.

Lenne PF, Wawrezinieck L, Conchonaud F, Wurtz O, Boned A, Guo XJ, Rigneault H, He HT, Marguet D. 2006. Dynamic molecular confinement in the plasma membrane by microdomains and the cytoskeleton meshwork. EMBO J 25: 3245-3256.

Levi V, Gratton E. 2007. Exploring dynamics in living cells by tracking single particles. Cell Biochem Biophys 48: $1-15$.

Levi V, Ruan QQ, Gratton E. 2005. 3-D particle tracking in a two-photon microscope: Application to the study of molecular dynamics in cells, Biophys J 88: 2919-2928.

Levi V, Gelfand VI, Serpinskaya AS, Gratton E. 2006a. Melanosomes transported by myosin-V in Xenopus melanophores perform slow $35 \mathrm{~nm}$ steps. Biophys J 90: L7-L9.
Fluorescence Techniques to Study Lipid Dynamics

Levi V, Serpinskaya AS, Gratton E, Gelfand V. 2006b. Organelle transport along microtubules in Xenopus melanophores: Evidence for cooperation between multiple motors. Biophys J 90: 318-327.

Lidke DS, Nagy P, Barisas BG, Heintzmann R, Post JN, Lidke KA, Clayton AHA, Arndt-Jovin DJ, Jovin TM. 2003. Imaging molecular interactions in cells by dynamic and static fluorescence anisotropy (rFLIM and emFRET). Biochem Soc Trans 31: 1020-1027.

Lingwood D, Ries J, Schwille P, Simons K. 2008. Plasma membranes are poised for activation of raft phase coalescence at physiological temperature. Proc Natl Acad Sci 105: 10005-10010.

Lippincott-Schwartz J, Snapp E, Kenworthy A. 2001. Studying protein dynamics in living cells. Nat Cell Biol 2: 444-456.

Lippincott-Schwartz J, Altan-Bonnet N, Patterson GH. 2003. Photobleaching and photoactivation: Following protein dynamics in living cells. Nat Cell Biol Suppl: S7-S14.

Loman A, Dertinger T, Koberling F, Enderlein J. 2008. Comparison of optical saturation effects in conventional and dual-focus fluorescence correlation spectroscopy. Chem Phys Lett 459: 18-21.

Lommerse PHM, Spaink HP, Schmidt T. 2004. In vivo plasma membrane organization: Results of biophysical approaches. Biochim Biophys Acta 1664: 119-131.

Machan R, Hof M. 2010. Lipid diffusion in planar membranes investigated by fluorescence correlation spectroscopy. Biochim Biophys Acta 1798: 1377-1391.

Magde D, Elson EL, Webb WW. 1974. Fluorescence correlation spectroscopy. 2. Experimental realization. Biopolymers 13: 29-61.

Magde D, Webb WW, Elson E. 1972. Thermodynamic fluctuations in a reacting system-Measurement by fluorescence correlation spectroscopy. Phys Rev Lett 29: 705.

Magde D, Webb WW, Elson EL. 1978. Fluorescence correlation spectroscopy. 3. Uniform translation and laminarflow. Biopolymers 17: 361-376.

Marks DL, Bittman R, Pagano RE. 2008. Use of Bodipylabeled sphingolipid and cholesterol analogs to examine membrane microdomains in cells. Histochem Cell Biol 130: 819-832.

Meder D, Moreno MJ, Verkade P, Vaz WLC, Simons K. 2006. Phase coexistence and connectivity in the apical membrane of polarized epithelial cells. Proc Natl Acad Sci 103: $329-334$.

Merrifield CJ, Perrais D, Zenisek D. 2005. Coupling between clathrin-coated-pit invagination, cortactin recruitment, and membrane scission observed in live cells. Cell 121: 593-606.

Merrifield CJ, Feldman ME, Wan L, Almers W. 2002. Imaging actin and dynamin recruitment during invagination of single clathrin-coated pits. Nat Cell Biol 4: 691-698.

Meseth U, Wohland T, Rigler R, Vogel H. 1999. Resolution of fluorescence correlation measurements. Biophys J 76: 1619-1631.

Middlebrook JL, Dorland RB. 1984. Bacterial toxins-Cellular mechanisms of action. Microbiological Rev 48: $199-221$. 
Mikhalyov I, Gretskaya N, Johansson LBA. 2009. Fluorescent BODIPY-labelled $\mathrm{G}_{\mathrm{M} 1}$ gangliosides designed for exploring lipid membrane properties and specific membrane-target interactions. Chem Phys Lipids 159: 38-44.

Mills JD, Stone JR, Rubin DG, Melon DE, Okonkwo DO, Periasamy A, Helm GA. 2003. Illuminating protein interactions in tissue using confocal and two-photon excitation fluorescent resonance energy transfer microscopy. J Biomed Opt 8: 347-356.

Miyawaki A, Tsien RY. 2000. Monitoring protein conformations and interactions by fluorescence resonance energy transfer between mutants of green fluorescent protein. Appl Chim Genes Hybrid Prot Pt B 327: 472-500.

Mueller BK, Zaychikov E, Brauchle C, Lamb DC. 2005. Pulsed interleaved excitation. Biophys J 89: 3508-3522.

Mütze J, Ohrt T, Schwille P. 2011. Fluorescence correlation spectroscopy in vivo. Laser Photon Rev 5: 52-67.

Nagamatsu S, Ohara-Imaizumi M. 2008. Imaging exocytosis of single insulin secretory granules with TIRF microscopy. Methods Mol Biol 440: 259-268.

Nicolau DV, Burrage K, Parton RG, Hancock JF. 2006. Identifying optimal lipid raft characteristics required to promote nanoscale protein-protein interactions on the plasma membrane. Mol Cell Biol 26: 313-323.

Niv H, Gutman O, Henis YI, Kloog Y. 1999. Membrane interactions of a constitutively active GFP-Ki-Ras 4B and their role in signaling-Evidence from lateral mobility studies. J Biol Chem 274: 1606-1613.

Niv H, Gutman O, Kloog Y, Henis YI. 2002. Activated K-Ras and $\mathrm{H}$-Ras display different interactions with saturable nonraft sites at the surface of live cells. J Cell Biol 157: $865-872$.

Ohara-Imaizumi M, Aoyagi K, Akimoto Y, Nakamichi Y, Nishiwaki C, Kawakami H, Nagamatsu S. 2009. Imaging exocytosis of single glucagon-like peptide- 1 containing granules in a murine enteroendocrine cell line with total internal reflection fluorescent microscopy. Biochem Biophys Res Comm 390: 16-20.

Oreopoulos J, Yip CM. 2009. Probing membrane order and topography in supported lipid bilayers by combined polarized total internal reflection fluorescence-atomic force microscopy. Biophys J 96: 1970-1984.

Parasassi T, Gratton E, Yu WM, Wilson P, Levi M. 1997. Twophoton fluorescence microscopy of Laurdan generalized polarization domains in model and natural membranes. Biophys J 72: 2413-2429.

Petersen NO. 1986. Scanning fluorescence correlation spectroscopy. 1. Theory and simulation of aggregation measurements. Biophys J 49: 809-815.

Petersen NO, Johnson DC, Schlesinger MJ. 1986. Scanning fluorescence correlation spectroscopy. 2. Application to virus glycoprotein aggregation. Biophys J 49: 817-820.

Petrasek Z, Schwille P. 2008. Precise measurement of diffusion coefficients using scanning fluorescence correlation spectroscopy. Biophys J 94: 1437-1448.

Petrasek Z, Ries J, Schwille P. 2010. Scanning FCS for the characterization of protein dynamics in live cells. Meth Enzymol 472: 317-343.

Petrov E, Schwille P. 2007. State of the art and novel trends in fluorescence correlation spectroscopy. In Standardization and quality assurance in fluorescence measurements II (ed. Resch-Genger U), pp. 145-197. Springer-Verlag, Berlin.

Phair RD, Gorski SA, Misteli T. 2004. Measurement of dynamic protein binding to chromatin in vivo, using photobleaching microscopy. Methods Enzymol 375: 393-414.

Pinaud F, Michalet X, Iyer G, Margeat E, Moore HP, Weiss S. 2009. Dynamic partitioning of a glycosyl-phosphatidylinositol-anchored protein in glycosphingolipid-rich microdomains imaged by single-quantum dot tracking. Traffic 10: 691-712.

Piston DW, Kremers GJ. 2007. Fluorescent protein FRET: The good, the bad and the ugly. Trends Biochem Sci 32: 407-414.

Ragan T, Huang HD, So P, Gratton E. 2006. 3D particle tracking on a two-photon microscope. J Fluorescence 16: $325-336$.

Ries J, Schwille P. 2006. Studying slow membrane dynamics with continuous wave scanning fluorescence correlation spectroscopy. Biophys J 91: 1915-1924.

Ries J, Chiantia S, Schwille P. 2009b. Accurate determination of membrane dynamics with line-scan FCS. Biophys J 96: 1999-2008.

Ries J, Petrov EP, Schwille P. 2008. Total internal reflection fluorescence correlation spectroscopy: Effects of lateral diffusion and surface-generated fluorescence. Biophys $J$ 95: 390-399.

Ries J, Yu SR, Burkhardt M, Brand M, Schwille P. 2009a. Modular scanning FCS quantifies receptor-ligand interactions in living multicellular organisms. Nat Methods 6: U643-U631.

Rigler R, Mets U, Widengren J, Kask P. 1993. Fluorescence correlation spectroscopy with high count rate and lowbackground-Analysis of translational diffusion. Euro Biophys J Biophys Lett 22: 169-175.

Rotblat B, Prior IA, Muncke C, Parton RG, Kloog Y, Henis YI, Hancock JF. 2004. Three separable domains regulate GTP-dependent association of H-ras with the plasma membrane. Mol Cell Biol 24: 6799-6810.

Roy S, Plowman S, Rotblat B, Prior IA, Muncke C, Grainger S, Parton RG, Henis YI, Kloog Y, Hancock JF. 2005. Individual palmitoyl residues serve distinct roles in $\mathrm{H}$-ras trafficking, microlocalization, and signaling. Mol Cell Biol 25: 6722-6733.

Runnels LW, Scarlata SF. 1995. Theory and application of fluorescence homotransfer to melittin oligomerization. Biophys J 69: 1569-1583.

Rusinova E, Tretyachenko-Ladokhina V, Vele OE, Senear DF, Ross JBA. 2002. Alexa and Oregon Green dyes as fluorescence anisotropy probes for measuring protein-protein and protein-nucleic acid interactions. Anal Biochem 308: $18-25$.

Sahoo H, Roccatano D, Hennig A, Nau WM. 2007. A 10 -angstrom spectroscopic ruler applied to short polyprolines. J Am Chem Soc 129: 9762-9772.

Sako Y, Minoghchi S, Yanagida T. 2000. Single-molecule imaging of EGFR signalling on the surface of living cells. Nat Cell Biol 2: 168-172.

Saxton MJ. 1994a. Anomalous diffusion due to obstaclesA Monte Carlo study. Biophys J 66: 394-401. 
Saxton MJ. 1994b. Single-particle tracking-Models of directed transport. Biophys J 67: 2110-2119.

Saxton MJ. 1995. Single-tracking-effects of corrals. Biophys J 69: $389-398$.

Saxton MJ. 1996a. Anomalous diffusion due to binding: A Monte Carlo study, Biophys J 70: 1250-1262.

Saxton MJ. 1996b. Single-particle tracking: New methods of data analysis, Biophys J 70: TU415-TU415.

Saxton MJ. 1997. Single-particle tracking: The distribution of diffusion coefficients. Biophys J 72: 1744-1753.

Saxton MJ, Jacobson K. 1997. Single-particle tracking: Applications to membrane dynamics. Annu Rev Biophys Biomol Struct 26: 373-399.

Scherfeld D, Kahya N, Schwille P. 2003. Lipid dynamics and domain formation in model membranes composed of ternary mixtures of unsaturated and saturated phosphatidylcholines and cholesterol. Biophys J 85: 37583768.

Schnapp BJ, Gelles J, Sheetz MP. 1988. Nanometer-scale measurements using video light-microscopy. Cell Motility Cytoskeleton 10: 47-53.

Schultz C, Neef AB, Gadella TW Jr, Goedhart J. 2010. Imaging lipids in living cells. Cold Spring Harb Protoc doi: 101101/pdbtop83.

Schutz GJ, Schindler H, Schmidt T. 1997. Single-molecule microscopy on model membranes reveals anomalous diffusion. Biophys J 73: 1073-1080.

Schutz GJ, Kada G, Pastushenko VP, Schindler H. 2000. Properties of lipid microdomains in a muscle cell membrane visualized by single molecule microscopy. $E M B O J$ 19: $892-901$.

Schwarzenbacher M, Kaltenbrunner M, Brameshuber M, Hesch C, Paster W, Weghuber J, Heise B, Sonnleitner A, Stockinger H, Schutz GJ. 2008. Micropatterning for quantitative analysis of protein-protein interactions in living cells. Nat Methods 5: 1053-1060.

Schwille P. 2003. TIR-FCS: Staying on the surface can sometimes be better. Biophys J 85: 2783-2784.

Schwille P, Heinze KG. 2001. Two-photon fluorescence cross-correlation spectroscopy. Chemphyschem 2: 269272.

Schwille P, Korlach J, Webb WW. 1999a. Fluorescence correlation spectroscopy with single-molecule sensitivity on cell and model membranes. Cytometry 36: 176-182.

Schwille P, MeyerAlmes FJ, Rigler R. 1997. Dual-color fluorescence cross-correlation spectroscopy for multicomponent diffusional analysis in solution. Biophys $J$ 72: $1878-1886$.

Schwille P, Haupts U, Maiti S, Webb WW. 1999b. Molecular dynamics in living cells observed by fluorescence correlation spectroscopy with one- and two-photon excitation. Biophys J 77: 2251-2265.

Schwille P, Heinze K, Dittrich P, Haustein E. 2009. Two-photon fluorescence correlation spectroscopy. In Biomedical optical imaging (ed. Fujimoto JG, Farkas D). Oxford University Press, Oxford.

Sharma P, Varma R, Sarasij RC, Ira K, Gousset G, Krishnamoorthy M, Rao S, Mayor S. 2004. Nanoscale organization of multiple GPI-anchored proteins in living cell membranes. Cell 116: 577-589.
Sheetz MP, Kuo SC. 1993. Tracking nanometer movements of single motor molecules. Meth Cell Biol 39: 129-136.

Sheppard CJR, Wilson T.1979. Effect of spherical-aberration on the imaging properties of scanning optical microscopes. Appl Opt 18: 1058-1063.

Shvartsman DE, Gutman O, Tietz A, Henis. 2006. Cyclodextrins but not compactin inhibit the lateral diffusion of membrane proteins independent of cholesterol. Traffic 7: 917-926.

Snapp EL, Altan N, Lippincott-Schwartz J. 2003. Measuring protein mobility by photobleaching GFP chimeras in living cells. Curr Protoc Cell Biol 21: 2121.

Sohn HW, Tolar P, Brzostowski J, Pierce SK. 2010. A method for analyzing protein-protein interactions in the plasma membrane of live B cells by fluorescence resonance energy transfer imaging as acquired by total internal reflection fluorescence microscopy. Methods Mol Biol 591: $159-183$.

Soumpasis DM. 1983. Theoretical analysis of fluorescence photobleaching recovery experiments. Biophysical J 41: 95-97.

Spandl J, White DJ, Peychl J, Thiele C. 2009. Live cell multicolor imaging of lipid droplets with a new dye, LD540. Traffic 10: 1579-1584.

Sprague BL, Pego RL, Stavreva DA, McNally JG. 2004. Analysis of binding reactions by fluorescence recovery after photobleaching. Biophys J 86: 3473-3495.

Sprague BL, McNally JG. 2005. FRAP analysis of binding: Proper and fitting. Trends in Cell Biol 15: 84-91.

Sum AK, Faller R, de Pablo JJ. 2003. Molecular simulation study of phospholipid bilayers and insights of the interactions with disaccharides. Biophys J 85: 2830-2844.

Teramura Y, Ichinose J, Takagi H, Nishida K, Yanagida T, Sako Y. 2006. Single-molecule analysis of epidermal growth factor binding on the surface of living cells. EMBO J 25: 4215-4222.

Thiele C, Spandl J. 2008. Cell biology of lipid droplets. Curr Opin Cell Biol 20: 378-385.

Tomishige M, Sako Y, Kusumi A. 1998. Regulation mechanism of the lateral diffusion of band 3 in erythrocyte membranes by the membrane skeleton. J Cell Biol 142: 989-1000.

Toprak E, Balci H, Blehm BH, Selvin PR. 2007. Threedimensional particle tracking via bifocal imaging. Nano Lett 7: 2043-2045.

Tyteca D, D’Auria L, Van Der Smissen P, Medts T, Carpentier S, Monbaliu JC, de Diesbach P, Courtoy PJ. 2010. Three unrelated sphingomyelin analogs spontaneously cluster into plasma membrane micrometric domains. Biochim Biophys Acta 1798: 909-927.

Vacha R, Siu SWI, Petrov M, Bockmann RA, Barucha-Kraszewska J, Jurkiewicz P, Hof M, Berkowitz ML, Jungwirth P. 2009. Effects of alkali cations and halide anions on the DOPC lipid membrane. J Phys Chem A 113: $7235-7243$.

van den Bogaart G, Hermans N, Krasnikov V, de Vries AH, Poolman B. 2007. On the decrease in lateral mobility of phospholipids by sugars. Biophys J 92: 1598-1605.

Varma R, Mayor S. 1998. GPI-anchored proteins are organized in submicron domains at the cell surface. Nature 394: $798-801$. 
E. Sezgin and P. Schwille

Veatch SL, Keller SL. 2002. Organization in lipid membranes containing cholesterol. Phys Rev Lett 89: 268101-268104.

Veatch SL, Keller SL. 2003. Separation of liquid phases in giant vesicles of ternary mixtures of phospholipids and cholesterol. Biophys J 85: 3074-3083.

Wallrabe H, Stanley M, Periasamy A, Barroso M. 2003. Oneand two-photon fluorescence resonance energy transfer microscopy to establish a clustered distribution of receptor-ligand complexes in endocytic membranes. J Biomed Opt 8: 339-346.

Wawrezinieck L, Rigneault H, Marguet D, Lenne PF. 2005. Fluorescence correlation spectroscopy diffusion laws to probe the submicron cell membrane organization. Biophys J 89: 4029-4042.

Weidemann T, Wachsmuth M, Tewes M, Rippe K, Langowski J. 2002. Analysis of ligand binding by two-colour fluorescence cross-correlation spectroscopy. Single Mol 3: $49-61$.

Weiss M. 2004. Challenges and artifacts in quantitative photobleaching experiments. Traffic 5: 662-671.

Wenger J, Conchonaud F, Dintinger J, Wawrezinieck L, Ebbesen TW, Rigneault H, Marguet D, Lenne PF. 2007. Diffusion analysis within single nanometric apertures reveals the ultrafine cell membrane organization. Biophys J 92: 913-919.

Wolf DE. 1989. Designing, building, and using a fluorescence recovery after photobleaching instrument. In Methods in cell biology (ed. Taylor DL, Wang Y-L), pp. 271-332. Academic Press, New York.
Worch R, Bokel C, Hofinger S, Schwille P, Weidemann T 2010. Focus on composition and interaction potential of single-pass transmembrane domains. Proteomics 10: 4196-4208.

Wouters FS, Bastiaens PIH, Wirtz KWA, Jovin TM. 1998. FRET microscopy demonstrates molecular association of non-specific lipid transfer protein (nsL-TP) with fatty acid oxidation enzymes in peroxisomes. EMBO $\mathrm{J}$ 17: 7179-7189.

Yildiz A, Forkey JN, McKinney SA, Ha T, Goldman YE, Selvin PR. 2003. Myosin V walks hand-over-hand: Single fluorophore imaging with $1.5-\mathrm{nm}$ localization. Science 300: 2061-2065.

Yildiz A, Park H, Safer D, Yang ZH, Chen LQ, Selvin PR, Sweeney HL. 2004a. Myosin VI steps via a handover-hand mechanism with its lever arm undergoing fluctuations when attached to actin. J Biol Chem 279: 37223-37226.

Yildiz A, Tomishige M, Vale RD, Selvin PR. 2004b. Kinesin walks hand-over-hand. Science 303: 676-678.

Yu CX, Hale J, Ritchie K, Prasad NK, Irudayaraj J. 2009. Receptor overexpression or inhibition alters cell surface dynamics of EGF-EGFR interaction: New insights from real-time single molecule analysis. Biochem Biophys Res Commun 378: 376-382.

Yu SR, Burkhardt M, Nowak M, Ries J, Petrasek Z, Scholpp S, Schwille P, Brand M. 2009. Fgf8 morphogen gradient forms by a source-sink mechanism with freely diffusing molecules. Nature 461: U533-U100. 


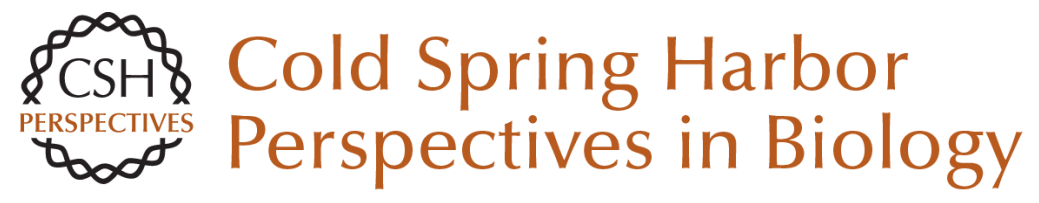

\section{Fluorescence Techniques to Study Lipid Dynamics}

Erdinc Sezgin and Petra Schwille

Cold Spring Harb Perspect Biol 2011; doi: 10.1101/cshperspect.a009803 originally published online June 13,2011

Subject Collection The Biology of Lipids

Role of Lipids in Virus Replication Maier Lorizate and Hans-Georg Kräusslich

Model Answers to Lipid Membrane Questions Ole G. Mouritsen

\section{Glycosphingolipid Functions} Clifford A. Lingwood

Regulation of Cholesterol and Fatty Acid Synthesis

Jin Ye and Russell A. DeBose-Boyd

Lipid-Mediated Endocytosis

Helge Ewers and Ari Helenius

Fluorescence Techniques to Study Lipid

Dynamics

Erdinc Sezgin and Petra Schwille

Lysosomal Lipid Storage Diseases

Heike Schulze and Konrad Sandhoff

Distribution and Functions of Sterols and Sphingolipids

J. Thomas Hannich, Kyohei Umebayashi and Howard Riezman
Membrane Organization and Lipid Rafts Kai Simons and Julio L. Sampaio

Shotgun Lipidomics on High Resolution Mass

Spectrometers

Dominik Schwudke, Kai Schuhmann, Ronny

Herzog, et al.

Glycosphingolipid Functions Clifford A. Lingwood

Phosphoinositides in Cell Architecture Annette Shewan, Dennis J. Eastburn and Keith Mostov

Synthesis and Biosynthetic Trafficking of Membrane Lipids Tomas Blom, Pentti Somerharju and Elina Ikonen

Lipid Polymorphisms and Membrane Shape Vadim A. Frolov, Anna V. Shnyrova and Joshua Zimmerberg

Specificity of Intramembrane Protein-Lipid Interactions

Francesc-Xabier Contreras, Andreas Max Ernst, Felix Wieland, et al.

Dynamic Transbilayer Lipid Asymmetry Gerrit van Meer

For additional articles in this collection, see http://cshperspectives.cshlp.org/cgi/collection/

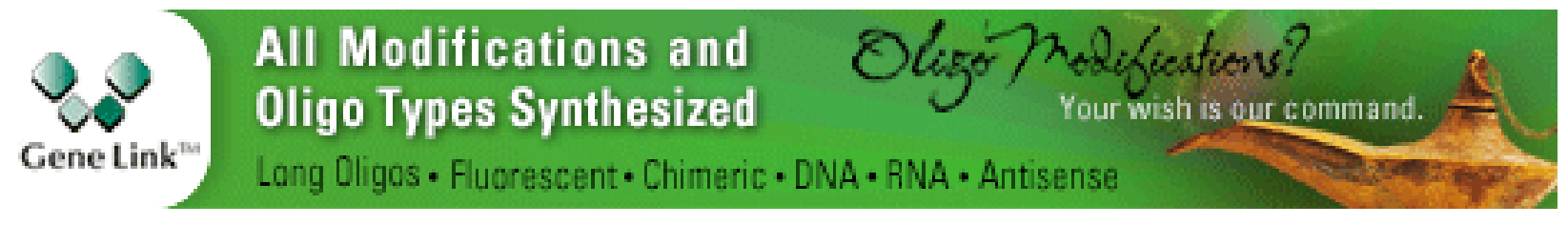

Copyright @ 2011 Cold Spring Harbor Laboratory Press; all rights reserved 